\title{
Composition of Irreducible Morphisms in Quasi-Tubes
}

\author{
Claudia CHAIO \\ Departamento de Matemática, Facultad de Ciencias Exactas y Naturales, \\ Funes 3350, Universidad Nacional de Mar del Plata, 7600 Mar del Plata, Argentina \\ E-mail: claudia.chaio@gmail.com \\ Piotr MALICKI \\ Faculty of Mathematics and Computer Science, Nicolaus Copernicus University, \\ Chopina 12/18, 87-100 Toruń, Poland \\ E-mail: pmalicki@mat.umk.pl
}

\begin{abstract}
We study the composition of irreducible morphisms between indecomposable modules lying in quasi-tubes of the Auslander-Reiten quivers of artin algebras in relation with the powers of the radical of their module category.
\end{abstract}

Keywords Irreducible morphism, Radical, Quasi-tube, Auslander-Reiten quiver, Selfinjective algebra

MR(2000) Subject Classification 16G70, 16G20, 16E10

\section{Introduction and the main results}

Throughout this paper, by an algebra we mean an artin algebra over a fixed commutative artin ring $R$. We denote by $\bmod A$ the category of finitely generated right $A$-modules and by ind $A$ the full subcategory of $\bmod A$ consisting of one representative of each isomorphism class of indecomposable $A$-modules.

We denote the radical of the module category $\bmod A$ by $\Re_{A}$. We recall that, for $X, Y \in$ ind $A$ the ideal $\Re_{A}(X, Y)$ is the set of all non-isomorphisms between $X$ and $Y$. Inductively, the powers of $\Re_{A}(X, Y)$ are defined. By $\Re_{A}^{\infty}(X, Y)$ we denote the intersection of all powers $\Re_{A}^{i}(X, Y)$ of $\Re_{A}(X, Y)$ with $i \geq 1$.

There is a close relationship between irreducible morphisms and the powers of the radical of its module category. In [?], Bautista proved that a morphism $f: X \rightarrow Y$ between indecomposable modules $X$ and $Y$ in $\bmod A$ is irreducible if and only if $f \in$ $\Re_{A}(X, Y) \backslash \Re_{A}^{2}(X, Y)$. This was generalized by Igusa and Todorov in [?, Theorem 13.3] where they proved that, for a sectional path

$$
X_{0} \stackrel{f_{1}}{\longrightarrow} X_{1} \stackrel{f_{2}}{\longrightarrow} \cdots \stackrel{f_{n-1}}{\longrightarrow} X_{n-1} \stackrel{f_{n}}{\longrightarrow} X_{n}
$$

of irreducible morphisms between indecomposable $A$-modules we have that their composition $f_{n} \ldots f_{2} f_{1} \in \Re_{A}^{n}\left(X_{0}, X_{n}\right) \backslash \Re_{A}^{n+1}\left(X_{0}, X_{n}\right)$.

The first author acknowledge partial support from CONICET and Universidad Nacional de Mar del Plata. The second named author gratefully acknowledge support from the research grant DEC2011/02/A/ST1/00216 of the Polish National Science Center. Moreover, authors was supported through the programme "Research in Pairs" by the Mathematisches Forschungsinstitut Oberwolfach in 2014. 
We denote by $\Gamma_{A}$ the Auslander-Reiten quiver of $A$, and by $\tau_{A}$ and $\tau_{A}^{-1}$ the AuslanderReiten translations $D \operatorname{Tr}$ and $\operatorname{Tr} D$, respectively. Recall that $\Gamma_{A}$ is a valued translation quiver defined as follows: the vertices of $\Gamma_{A}$ are the isomorphism classes $[X]$ of modules $X$ in ind $A$, we put an arrow from $[X] \rightarrow[Y]$ in $\Gamma_{A}$ if there is an irreducible morphism from $X$ to $Y$ in $\bmod A$. The valuation $\left(d_{X Y}, d_{X Y}^{\prime}\right)$ of an arrow $[X] \rightarrow[Y]$ in $\Gamma_{A}$ is defined such that $d_{X Y}$ is the multiplicity of $Y$ in the codomain of the minimal left almost split morphism for $X$ and $d_{X Y}^{\prime}$ is the multiplicity of $X$ in the domain of the minimal right almost split morphism for $Y$. We shall not distinguish between an indecomposable $A$ module and the vertex of $\Gamma_{A}$ corresponding to it. Moreover, the valuation $(1,1)$ of an arrow in $\Gamma_{A}$ will be omitted and we will say that a component $\Gamma$ of $\Gamma_{A}$ has trivial valuation if all arrows in $\Gamma$ have valuation $(1,1)$.

By a component of $\Gamma_{A}$ we mean a connected component of the quiver $\Gamma_{A}$. In general, the Auslander-Reiten quiver $\Gamma_{A}$ describes only the quotient category $\bmod A / \Re_{A}^{\infty}$.

An important research direction towards understanding the structure of module categories is the study of compositions of irreducible morphisms between indecomposable modules.

In [?], S. Liu introduced the notion of degree of an irreducible morphism of modules (??) and using such a concept he described the shapes of the components of the Auslander-Reiten quivers of algebras of infinite representation type. Liu also, studied the composition of irreducible morphisms between indecomposable modules, generalizing Igusa and Todorov result concerning sectional paths. More precisely, Liu defined the notion of pre-sectional path (??) and proved that if

$$
X_{0} \longrightarrow X_{1} \longrightarrow \ldots \longrightarrow X_{n-1} \longrightarrow X_{n}
$$

is a pre-sectional path then there are irreducible morphisms $g_{i}: X_{i-1} \longrightarrow X_{i}$ for $i=$ $1, \ldots, n$, such that their composition $g_{n} \ldots g_{2} g_{1}$ lies in $\Re_{A}^{n}\left(X_{0}, X_{n}\right) \backslash \Re_{A}^{n+1}\left(X_{0}, X_{n}\right)$.

Recently, there has been many new results related to the subject of the composition of irreducible morphisms and their relation with the power of the radical of their module category. Most of them involving the concept of degree. For instance, see [?, ?, ?, ?, ?, $?, ?, ?, ?]$.

In [?], the authors looked at the general situation of when the composite of two irreducible morphisms is a non-zero morphism and lies in $\Re_{A}^{3}$ for $A$ an artin algebra. In particular, by [?] we are able to determine if a finite dimensional algebra over an algebraically closed field is of finite representation type by computing the degree of a finite number of irreducible morphisms. Moreover, in [?] whenever we deal with a representation-finite algebra, the minimal lower bound $m \geq 1$ such that $\Re_{A}^{m}$ vanishes, was given. This bound was determined in terms of the right and the left degree of irreducible morphisms, not depending on the maximal length of the indecomposable modules. This result was extended in [?] where the authors found the nilpotency of the radical of a module category for any artin algebra.

In [?], the authors studied the finiteness of degrees of irreducible morphisms between indecomposable modules lying in coherent almost cyclic components of Auslander-Reiten quivers of artin algebras.

In the representation theory of selfinjective algebras a prominent role played the components called quasi-tubes, whose stable parts are stable tubes. By general theory [?], [?], an infinite component $\Gamma$ of the Auslander-Reiten quiver $\Gamma_{A}$ of a selfinjective algebra $A$ is a quasi-tube if and only if $\Gamma$ contains an oriented cycle. The quasi-tubes occur 
in the Auslander-Reiten quivers of many selfinjective algebras, for example, for: the representation-infinite blocks of group algebras [?], [?], the representation-infinite tame algebras [?], the selfinjective algebras of wild canonical type [?], and the deformed preprojective algebras of generalized Dynkin type [?]. We also refer to the article [?] for the bound on the number of simple and projective modules in the quasi-tubes of the Auslander-Reiten quivers of finite dimensional selfinjective algebras over a field.

We would like to mention that the quasi-tubes occur also in the Auslander-Reiten quivers of the generalized multicoil algebras (see for instance [?, ?, ?, ?, ?, ?, ?, ?] for their structure and importance), which are obtained by sophisticated gluings of concealed canonical algebras using ten admissible algebra operations, generalizing the coil operations introduced in [?].

In this paper we are interested in the composition of irreducible morphisms between indecomposable modules lying in quasi-tubes of Auslander-Reiten quivers of artin algebras. In particular, we study the composition of irreducible morphisms between indecomposable modules in selfinjective algebras (where projective are also injective $A$-modules) and tubes in a general sense.

Let $A$ be an artin algebra. In order to formulate one of our main results we define a special type of full translation subquiver of $\Gamma_{A}$. A full translation subquiver of $\Gamma_{A}$ of the form

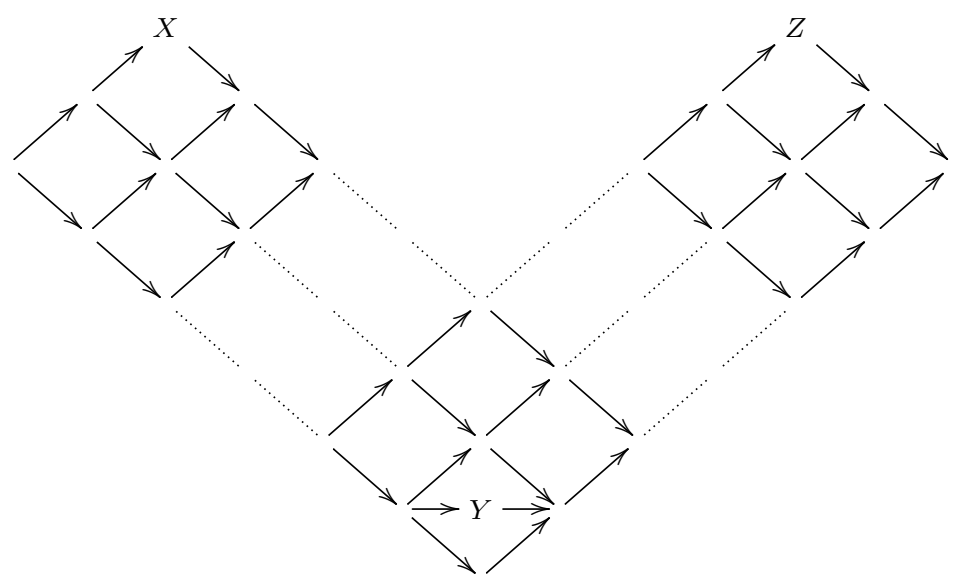

with $X, Y$ and $Z$ indecomposable projective-injective $A$-modules is said to be a special configuration of modules.

The main results proven in this work are the following theorems.

Theorem A. Let $A$ be a selfinjective artin algebra and $\Gamma$ an infinite component of $\Gamma_{A}$ without special configurations of modules and containing an oriented cycle. Let

$$
X_{1} \stackrel{f_{1}}{\longrightarrow} X_{2} \stackrel{f_{2}}{\longrightarrow} \cdots \stackrel{f_{n-1}}{\longrightarrow} X_{n} \stackrel{f_{n}}{\longrightarrow} X_{n+1}
$$

be a path of irreducible morphisms with $X_{i} \in \Gamma$ for $i=1, \ldots, n+1$. Then, $f_{n} \ldots f_{1} \in$ $\Re_{A}^{n+1}\left(X_{1}, X_{n+1}\right)$ if and only if $f_{n} \ldots f_{1} \in \Re_{A}^{\infty}\left(X_{1}, X_{n+1}\right)$.

Theorem B. Let $A$ be an artin algebra and $\Gamma$ a tube in $\Gamma_{A}$. Let $h_{i}: X_{i} \rightarrow X_{i+1}$ be $n$ irreducible morphisms with $X_{i} \in \Gamma$ for $i=1, \ldots, n$. Then, $0 \neq h_{n} \ldots h_{1} \in \Re_{A}^{n+1}\left(X_{1}, X_{n+1}\right)$ if and only if $0 \neq h_{n} \ldots h_{1} \in \Re_{A}^{\infty}\left(X_{1}, X_{n+1}\right)$. 
For basic background on the representation theory of algebras we refer to [?], [?] and [?].

\section{Preliminaries}

2.1. Let $A$ be an algebra, $X, Y$ be the modules in ind $A$, and $f: X \rightarrow Y$ be an irreducible morphism in $\bmod A$. If $X$ is not injective, we shall denote by $\epsilon(X)$ the almost split sequence starting at $X$ and by $\alpha(X)$ the number of indecomposable direct summands of the middle term of $\epsilon(X)$.

2.2. Let $A$ be an algebra. Given $X, Y \in \bmod A$, the ideal $\Re_{A}(X, Y)$ is the set of all the morphisms $f: X \rightarrow Y$ such that, for each $M \in$ ind $A$, each $h: M \rightarrow X$ and each $h^{\prime}: Y \rightarrow M$ the composition $h^{\prime} f h$ is not an isomorphism. In particular, if $X, Y \in$ ind $A$ then $\Re_{A}(X, Y)$ is the set of all the morphisms $f: X \rightarrow Y$ which are not isomorphisms. Inductively, the powers of $\Re_{A}(X, Y)$ are defined. By $\Re_{A}^{\infty}(X, Y)$ we denote the intersection of all powers $\Re_{A}^{i}(X, Y)$ of $\Re_{A}(X, Y)$, with $i \geq 1$.

Next, we state the definition of degree of an irreducible morphism given by S. Liu in [?].

2.3. Let $A$ be an algebra and let $f: X \rightarrow Y$ be an irreducible morphism in $\bmod A$, with $X$ or $Y$ indecomposable. Following [?] the left degree $d_{l}(f)$ of $f$ is infinite, if for each integer $n \geq 1$, each module $Z \in \bmod A$ and each morphism $g \in \Re_{A}^{n}(Z, X) \backslash \Re_{A}^{n+1}(Z, X)$ we have that $f g \notin \Re_{A}^{n+2}(Z, Y)$. Otherwise, the left degree of $f$ is the smallest positive integer $m$ such that there is an $A$-module $Z$ and a morphism $g \in \Re_{A}^{m}(Z, X) \backslash \Re_{A}^{m+1}(Z, X)$ such that $f g \in \Re_{A}^{m+2}(Z, Y)$.

The right degree $d_{r}(f)$ of an irreducible morphism $f$ is dually defined.

2.4. Let $A$ be an algebra. By a path in $\Gamma_{A}$ we mean a sequence of irreducible morphisms between indecomposable modules $Y_{1} \rightarrow Y_{2} \rightarrow \cdots \rightarrow Y_{n-1} \rightarrow Y_{n}$, and by a non-zero path (zero-path) we mean that the composition of the irreducible morphisms of the path does not vanish (vanishes).

In [?], Bautista defined the notion of sectional paths. A path $Y_{1} \rightarrow Y_{2} \rightarrow \cdots \rightarrow Y_{n-1} \rightarrow$ $Y_{n}$ in $\Gamma_{A}$ is said to be sectional if for each $i=2, \ldots, n-1$ we have that $Y_{i+1} \not \tau_{A}{ }^{-1} Y_{i-1}$.

In [?], Liu generalized such a concept defining what he called a pre-sectional path. A path $Y_{1} \rightarrow Y_{2} \rightarrow \cdots \rightarrow Y_{n-1} \rightarrow Y_{n}$ in $\Gamma_{A}$ is said to be pre-sectional if, whenever $Y_{i-1}=\tau_{A} Y_{i+1}$ for $i=2, \ldots, n-1$ then $Y_{i-1} \oplus \tau_{A} Y_{i+1}$ is a summand of the domain of a right almost split morphism for $Y_{i}$, or equivalently, whenever $\tau_{A}^{-1} Y_{i-1}=Y_{i+1}$ implies that $\tau_{A}^{-1} Y_{i-1} \oplus Y_{i+1}$ is a summand of the codomain of a left almost split morphism for $Y_{i}$. Observe that any sectional path is a pre-sectional path.

Furthermore, in [?] Igusa and Todorov proved that if

$$
X_{0} \stackrel{f_{1}}{\longrightarrow} X_{1} \stackrel{f_{2}}{\longrightarrow} \cdots \stackrel{f_{n-1}}{\longrightarrow} X_{n-1} \stackrel{f_{n}}{\longrightarrow} X_{n}
$$

is a sectional path then the composition $f_{n} \ldots f_{1}: X_{0} \rightarrow X_{n}$ is such that $f_{n} \ldots f_{1} \in$ $\Re^{n}\left(X_{0}, X_{n}\right) \backslash \Re^{n+1}\left(X_{0}, X_{n}\right)$. In [?, Lemma 1.15], Liu extended the above result to presectional paths and proved that if $X_{0} \rightarrow X_{1} \rightarrow \cdots \rightarrow X_{n-1} \rightarrow X_{n}$ is a pre-sectional path then there are irreducible morphisms $f_{i}: X_{i} \rightarrow X_{i+1}$ for $i=0, \ldots, n-1$ such that $f_{n-1} \ldots f_{0} \in \Re^{n}\left(X_{0}, X_{n}\right) \backslash \Re^{n+1}\left(X_{0}, X_{n}\right)$. 
By a cycle in $\Gamma_{A}$ we mean a sequence of irreducible morphisms between indecomposable modules of the form $Y_{1} \rightarrow Y_{2} \rightarrow \cdots \rightarrow Y_{n-1} \rightarrow Y_{n} \rightarrow Y_{1}$.

2.5. Recall that if $\mathbb{A}_{\infty}$ is the quiver $0 \rightarrow 1 \rightarrow 2 \rightarrow \cdots$ (with trivial valuations $(1,1)$ ), then $\mathbb{Z} \mathbb{A}_{\infty}$ is the translation quiver of the form:

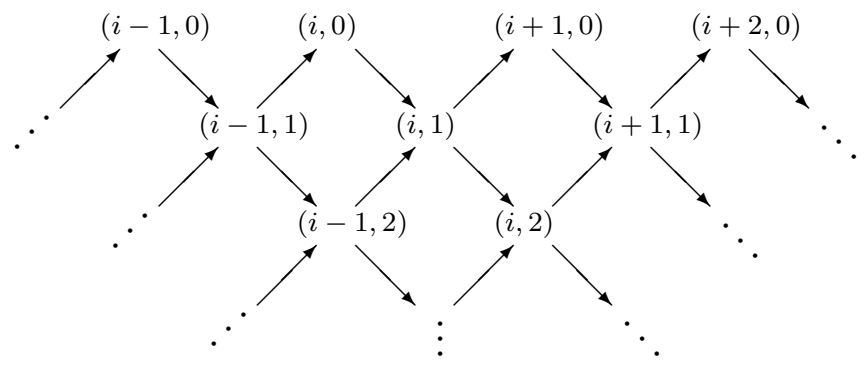

with $\tau(i, j)=(i-1, j)$ for $i \in \mathbb{Z}, j \in \mathbb{N}$. For $r \geq 1$, denote by $\mathbb{Z} \mathbb{A}_{\infty} /\left(\tau^{r}\right)$ the translation quiver $\Gamma$ obtained from $\mathbb{Z} \mathbb{A}_{\infty}$ by identifying each vertex $(i, j)$ of $\mathbb{Z} \mathbb{A}_{\infty}$ with the vertex $\tau^{r}(i, j)$ and each arrow $x \rightarrow y$ in $\mathbb{Z} \mathbb{A}_{\infty}$ with the arrow $\tau^{r} x \rightarrow \tau^{r} y$. The translation quiver of the form $\mathbb{Z} \mathbb{A}_{\infty} /\left(\tau^{r}\right)$ is called stable tube of rank $r$. The rank of a stable tube $\Gamma$ is the least positive integer $r$ such that $\tau^{r} x=x$ for all $x$ in $\Gamma$. The $\tau$-orbit of a stable tube $\Gamma$ formed by all vertices having exactly one direct predecessor is said to be the mouth of $\Gamma$.

Let $(\Gamma, \tau)$ be a translation quiver with trivial valuations. For a vertex $X$ in $\Gamma$, called the pivot, we shall define two admissible operations [?] modifying $(\Gamma, \tau)$ to a new translation quiver $\left(\Gamma^{\prime}, \tau^{\prime}\right)$ depending on the shape of paths in $\Gamma$ starting from $X$.

(ad 1) Suppose that $\Gamma$ admits an infinite sectional path

$$
X=X_{0} \rightarrow X_{1} \rightarrow X_{2} \rightarrow \cdots
$$

starting at $X$, and assume that every sectional path in $\Gamma$ starting at $X$ is a subpath of the above path. For $t \geq 1$, let $\Gamma_{t}$ be the following translation quiver, isomorphic to the Auslander-Reiten quiver of the full $t \times t$ lower triangular matrix algebra,

We then let $\Gamma^{\prime}$ be the translation quiver having as vertices those of $\Gamma$, those of $\Gamma_{t}$, additional vertices $Z_{i j}$ and $X_{i}^{\prime}$ (where $i \geq 0,1 \leq j \leq t$ ) and having arrows as in the figure below

The translation $\tau^{\prime}$ of $\Gamma^{\prime}$ is defined as follows: $\tau^{\prime} Z_{i j}=Z_{i-1, j-1}$ if $i \geq 1, j \geq 2, \tau^{\prime} Z_{i 1}=X_{i-1}$ if $i \geq 1, \tau^{\prime} Z_{0 j}=Y_{j-1}$ if $j \geq 2, Z_{01}$ is projective, $\tau^{\prime} X_{0}^{\prime}=Y_{t}, \tau^{\prime} X_{i}^{\prime}=Z_{i-1, t}$ if $i \geq 1, \tau^{\prime}\left(\tau^{-1} X_{i}\right)=$ $X_{i}^{\prime}$ provided $X_{i}$ is not injective in $\Gamma$, otherwise $X_{i}^{\prime}$ is injective in $\Gamma^{\prime}$. For the remaining vertices of $\Gamma^{\prime}, \tau^{\prime}$ coincides with the translation of $\Gamma$, or $\Gamma_{t}$, respectively. If $t=0$, the new translation quiver $\Gamma^{\prime}$ is obtained from $\Gamma$ by inserting only the sectional path consisting of the vertices $X_{i}^{\prime}, i \geq 0$.

(ad 2) Suppose that $\Gamma$ admits two sectional paths starting at $X$, one infinite and the other finite with at least one arrow

$$
Y_{t} \leftarrow \cdots \leftarrow Y_{2} \leftarrow Y_{1} \leftarrow X=X_{0} \rightarrow X_{1} \rightarrow X_{2} \rightarrow \cdots
$$


such that any sectional path starting at $X$ is a subpath of one of these paths and $X_{0}$ is injective. Then $\Gamma^{\prime}$ is the translation quiver having as vertices those of $\Gamma$, additional vertices denoted by $X_{0}^{\prime}, Z_{i j}, X_{i}^{\prime}$ (where $i \geq 1,1 \leq j \leq t$ ), and having arrows as in the figure below

The translation $\tau^{\prime}$ of $\Gamma^{\prime}$ is defined as follows: $X_{0}^{\prime}$ is projective-injective, $\tau^{\prime} Z_{i j}=Z_{i-1, j-1}$ if $i \geq 2, j \geq 2, \tau^{\prime} Z_{i 1}=X_{i-1}$ if $i \geq 1, \tau^{\prime} Z_{1 j}=Y_{j-1}$ if $j \geq 2, \tau^{\prime} X_{i}^{\prime}=Z_{i-1, t}$ if $i \geq 2, \tau^{\prime} X_{1}^{\prime}=Y_{t}$, $\tau^{\prime}\left(\tau^{-1} X_{i}\right)=X_{i}^{\prime}$ provided $X_{i}$ is not injective in $\Gamma$, otherwise $X_{i}^{\prime}$ is injective in $\Gamma^{\prime}$. For the remaining vertices of $\Gamma^{\prime}, \tau^{\prime}$ coincides with the translation $\tau$ of $\Gamma$.

We denote by $\left(\right.$ ad $1^{*}$ ) and (ad $2^{*}$ ) the admissible operations dual to the admissible operations ( $\operatorname{ad} 1)$ and $(\operatorname{ad} 2)$, respectively.

A connected translation quiver $\Gamma$ is said to be a quasi-tube if $\Gamma$ can be obtained from a stable tube $\mathscr{T}=\mathbb{Z} \mathbb{A}_{\infty} /\left(\tau^{r}\right)$ by an iterated application of admissible operations $(\operatorname{ad} 1)$, $(\operatorname{ad} 2),\left(\operatorname{ad} 1^{*}\right)$ or $\left(\operatorname{ad} 2^{*}\right)$. A tube (in the sense of [?]) is a quasi-tube having the property that each admissible operation in the sequence defining it is of the form (ad 1) or (ad $\left.1^{*}\right)$, that is, it contains a cyclical path and its underlying topological space is homeomorphic to $S^{1} \times \mathbb{R}^{+}$, where $S^{1}$ is the unit circle and $\mathbb{R}^{+}$is the nonnegative real line. Finally, if we apply only operations of type (ad 1) (respectively, of type $\left.\left(\operatorname{ad} 1^{*}\right)\right)$, then such a quasitube $\Gamma$ is called a ray tube (respectively, a coray tube). Observe that a quasi-tube without injective (respectively, projective) vertices is a ray tube (respectively, a coray tube). A quasi-tube $\Gamma$ whose all non-stable vertices are projective-injective is said to be smooth.

The following proposition provides a characterization of quasi-tubes in the AuslanderReiten quivers of selfinjective artin algebras ([?, Theorem A], [?],[?]).

Proposition 2.6. Let $A$ be a selfinjective artin algebra and $\Gamma$ a component of $\Gamma_{A}$. The following statements are equivalent:

(a) $\Gamma$ is a quasi-tube.

(b) $\Gamma^{s}$ is a stable tube.

(c) $\Gamma$ contains an oriented cycle.

Here, $\Gamma^{s}$ denotes the stable part of $\Gamma$, obtained from $\Gamma$ by removing the projectiveinjective modules and the arrows attached to them.

Let $A$ be an algebra, and let $\mathscr{T}$ be a stable tube of $\Gamma_{A}$. Then $\mathscr{T}$ has two types of arrows: arrows pointing to infinity and arrows pointing to the mouth. Hence, for any module $Z$ lying in $\mathscr{T}$, there is a unique sectional path $X_{1} \rightarrow X_{2} \rightarrow \cdots \rightarrow X_{t}=Z$ in $\mathscr{T}$ with $X_{1}$ lying on the mouth of $\mathscr{T}$ (consisting of arrows pointing to infinity) and there is a unique sectional path $Z=Y_{1} \rightarrow Y_{2} \rightarrow \cdots \rightarrow Y_{t}$ with $Y_{t}$ lying on the mouth of $\mathscr{T}$ (consisting of arrows pointing to the mouth), and $t$ is called the quasi-length of $Z$ in $\mathscr{T}$, denoted by $\mathrm{ql}(Z)$. Now, let $\mathscr{C}$ be a smooth quasi-tube in $\Gamma_{A}$. Then the stable part $\mathscr{C}^{s}$ of $\mathscr{C}$ is a stable tube, and we may define the smooth quasi-length $\operatorname{sql}(X)$ of $X$ from $\mathscr{C}$ as follows:

$$
\operatorname{sql}(X)= \begin{cases}\mathrm{ql}(X) & \text { if } X \in \mathscr{C}^{s} \\ \mathrm{ql}\left(X^{+}\right) & \text {otherwise }\end{cases}
$$

where for $X \in \mathscr{C} \backslash \mathscr{C}^{s}, X^{+}$(respectively, $X^{-}$) denotes the immediate successor (respectively, immediate predecessor) of $X$ in $\mathscr{C}$. Note that, if $X \in \mathscr{C} \backslash \mathscr{C}^{s}$ then $\operatorname{sql}(X)=$ $\mathrm{ql}\left(X^{+}\right)=\mathrm{ql}\left(X^{-}\right)$. 
2.7. We recall that a component $\Gamma$ of $\Gamma_{A}$ is called almost cyclic if all but finitely many modules of $\Gamma$ lie on oriented cycles. Further, a component $\Gamma$ of $\Gamma_{A}$ is called coherent if conditions $(\mathrm{C} 1)$ and $(\mathrm{C} 2)$ are satisfied:

(C1) For each projective module $P$ in $\Gamma$ there is an infinite sectional path

$$
P=X_{1} \rightarrow X_{2} \rightarrow \cdots \rightarrow X_{i} \rightarrow X_{i+1} \rightarrow X_{i+2} \rightarrow \cdots
$$

(C2) For each injective module $I$ in $\Gamma$ there is an infinite sectional path

$$
\cdots \rightarrow Y_{j+2} \rightarrow Y_{j+1} \rightarrow Y_{j} \rightarrow \cdots \rightarrow Y_{2} \rightarrow Y_{1}=I .
$$

\section{The results}

We start this section recalling the definition of depth of a morphism given in [?].

Definition 3.1. Let $A$ be an artin algebra and $f: M \rightarrow N$ be a morphism in $\bmod A$. We say that the depth of $f$, denoted by $\operatorname{dp}(f)$, is infinite in case $f \in \Re_{A}^{\infty}(M, N)$; otherwise, is the integer $n \geq 0$ for which $f \in \Re_{A}^{n}(M, N)$ but $f \notin \Re_{A}^{n+1}(M, N)$.

For the convenience of the reader we state [?, Lemma 2.1] and [?, Proposition 2.2] which we will useful all through this paper. In fact, taking into account these results it is not hard to see that it is enough to study the irreducible morphisms satisfying the mesh relations of the components in consideration in order to have information on the irreducible morphisms of $\bmod A$.

Lemma 3.2. ([?, Lemma 2.1]) Let $A$ be an artin algebra and $\Gamma$ be a component of $\Gamma_{A}$ with trivial valuation. Let $h_{i}: X_{i} \rightarrow X_{i+1}$ be an irreducible morphism with $X_{i} \in \Gamma$, for $i=1, \ldots, n$. Then, for any choice of irreducible morphisms $f_{i}: X_{i} \rightarrow X_{i+1}$ we have that $h_{n} \ldots h_{1}=\delta f_{n} \ldots f_{1}+\mu$ with $\delta \in \operatorname{Aut}\left(X_{n+1}\right)$ and $\mu \in \Re_{A}^{n+1}\left(X_{1}, X_{n+1}\right)$.

Let $f: X \rightarrow Y$ be an irreducible morphism between indecomposable modules in $\bmod A$. We set

$$
\operatorname{Irr}(X, Y)=\Re_{A}(X, Y) / \Re_{A}^{2}(X, Y) .
$$

We recall that $\operatorname{Irr}(X, Y)$ is a $k_{X}-k_{Y}$-bimodule where $k_{X}=\operatorname{End}(X) / \Re_{A}(X, X)$ and $k_{Y}=\operatorname{End}(Y) / \Re_{A}(Y, Y)$. Moreover, $k_{Z}$ is a division ring whenever $Z$ is an indecomposable $A$-module.

Proposition 3.3. [?, Proposition 2.2] Let $A$ be an artin algebra and $X_{i} \in$ ind $A$ for $1 \leq i \leq n+1$. Assume that $\operatorname{dim}_{k_{X_{i}}} \operatorname{Hom}_{A}\left(X_{i}, X_{i+1}\right)=\operatorname{dim}_{k_{X_{i+1}}} \operatorname{Hom}_{A}\left(X_{i}, X_{i+1}\right)=1$, for $i=1, \ldots, n$. Then, the following conditions are equivalent:

(a) There are irreducible morphisms $f_{i}: X_{i} \rightarrow X_{i+1}$ in $\bmod A$, for $i=1, \cdots, n$ with $f_{n} \cdots f_{1} \notin \Re_{A}^{n+1}\left(X_{1}, X_{n+1}\right)$.

(b) Given any irreducible morphisms $h_{i}: X_{i} \rightarrow X_{i+1}$ in $\bmod A$, for $i=1, \cdots, n$, then $h_{n} \ldots h_{1} \notin \Re_{A}^{n+1}\left(X_{1}, X_{n+1}\right)$. 
We shall dedicate the first part of this paper to study the composition of irreducible morphisms lying in an exceptional wing. We observe that these mentioned wings appear in coherent almost cyclic Auslander-Reiten components (see [?]). We start given the definition of exceptional wings.

Definition 3.4. A full translation subquiver of $\Gamma_{A}$ of one of the forms
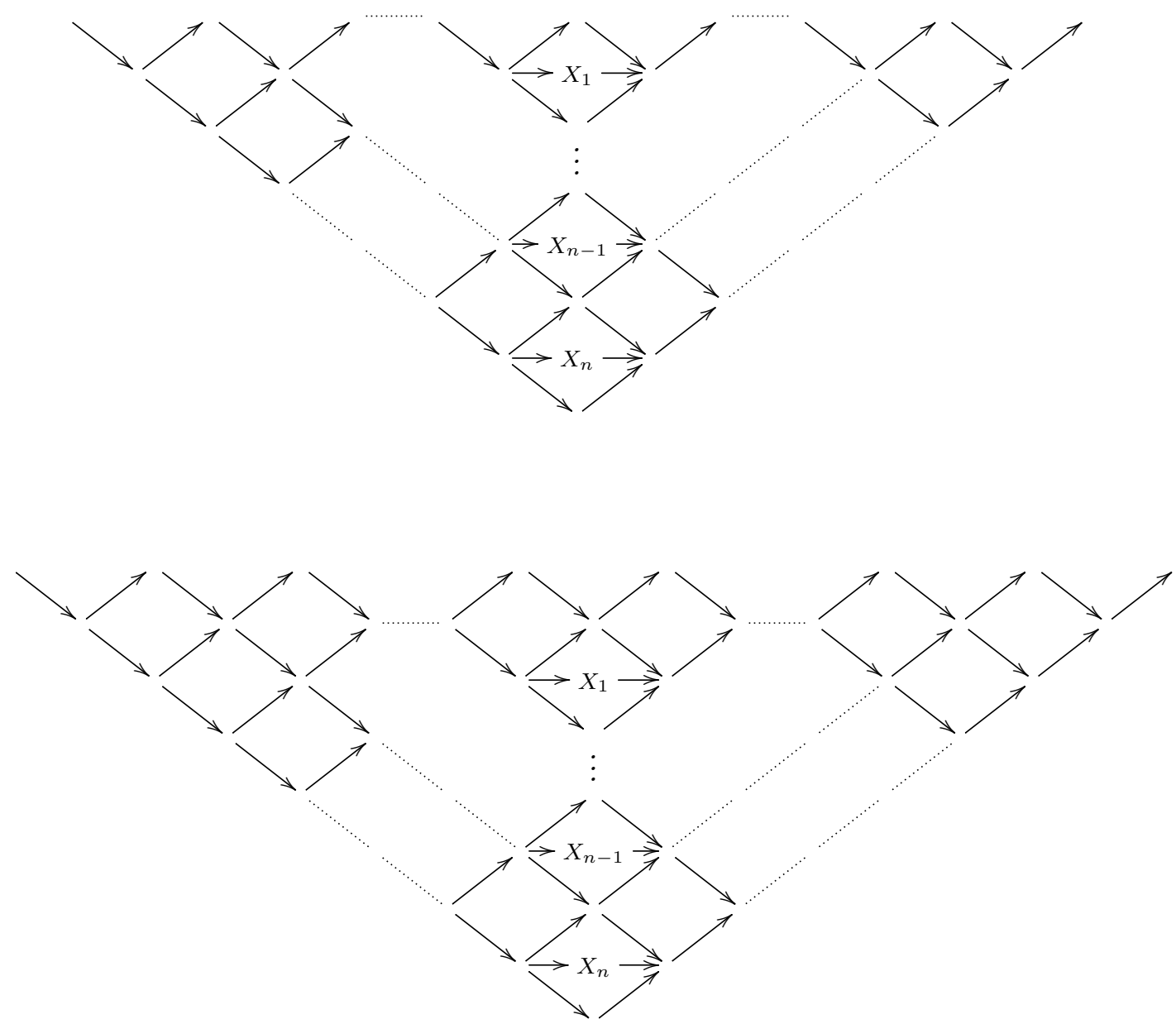

where $n \geq 1, X_{i}, 1 \leq i \leq n$ are indecomposable projective-injective modules, and $X_{n} \neq 0$; or a wing in the sense of Ringel (see [?])

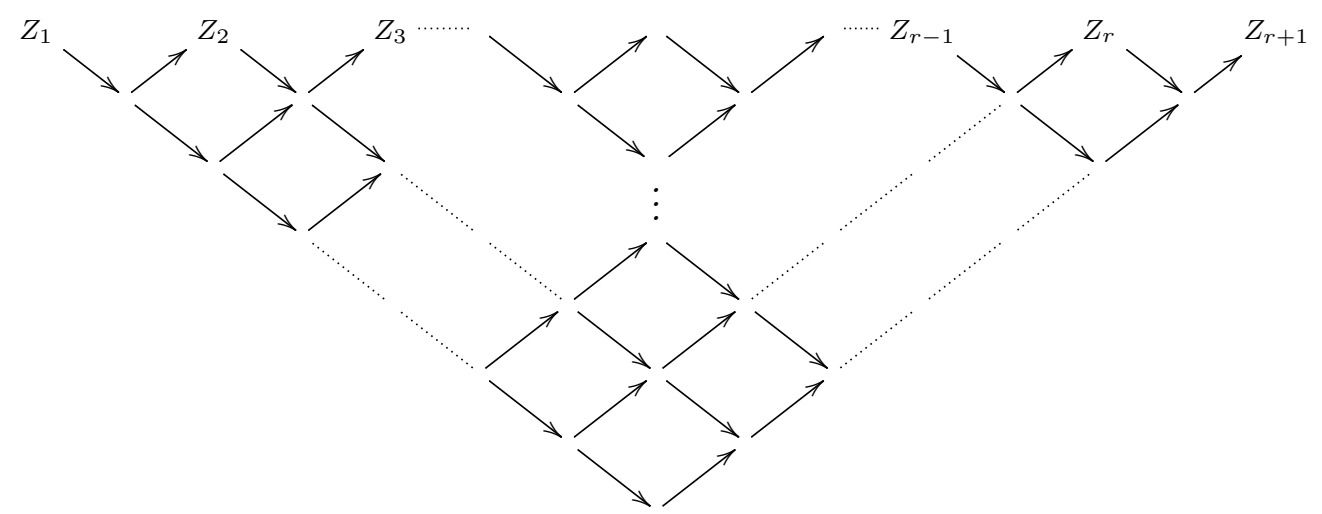


is said to be an exceptional wing. We denote it by $\mathcal{W}$. The two maximal sectional paths of $\mathcal{W}$ are called the borders of $\mathcal{W}$.

Definition 3.5. We say that a composition $\varphi_{n_{m}} \ldots \varphi_{n_{1}}$ of morphisms (irreducible morphisms, resp.) $\varphi_{n_{j}}$, for $j=1, \ldots, m$, in $\bmod A$ (in a component $\Gamma$, resp.) behaves well whenever $\operatorname{dp}\left(\varphi_{n_{j}}\right)=r_{j}$ with $r_{j} \geq 0$ then we have that $\operatorname{dp}\left(\varphi_{n_{m}} \ldots \varphi_{n_{1}}\right)=r_{m}+\cdots+r_{1}$.

We observe that for the proof of the converse of [?, Proposition 6.1] we do not need the hypothesis of $\Gamma$ been a component of $\Gamma_{A}$ satisfying $\alpha(\Gamma) \leq 2$ (the number of indecomposable direct summands of the middle term of all almost split sequence is less than or equal to 2). Such a hypothesis was only necessary for the other implication. In order to make this comment clear we shall include a proof of this fact in Lemma ??, Statement (a).

Next, we prove three technical lemmas which will allow us to study the composition of irreducible morphisms lying in an exceptional wing $\mathcal{W}$. More precisely, we shall prove that the composition of the irreducible morphisms in the borders of $\mathcal{W}$ behaves well.

Lemma 3.6. Let $A$ be an artin algebra, $\Gamma$ a component of $\Gamma_{A}, X_{i} \in \Gamma$ for $i=0, \ldots, n$ and $n \geq 1$. Let $f: X_{n} \rightarrow X_{n+1}$ be an irreducible morphism and assume that there is a configuration of almost split sequences as follows

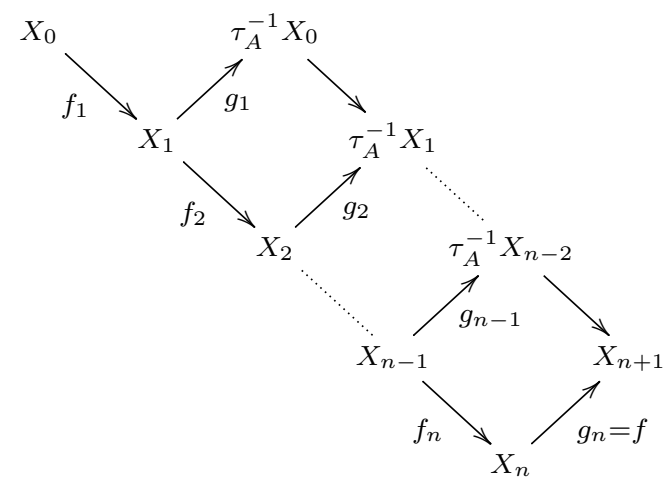

with $\alpha\left(X_{i}\right)=2$ for $i=1, \ldots, n-1, \alpha\left(X_{0}\right)=1$ and $f_{n} \ldots f_{1}$ a sectional path. Moreover, assume there is a morphism $\mu: X \rightarrow X_{n}$ with $X \in \Gamma$ such that $\operatorname{dp}(\mu)=m$ for some positive integer $m$ and $f \mu \in \Re_{A}^{m+2}\left(X, X_{n+1}\right)$. Then,

(a) The left degree of $f$ is $n$ and $m \geq n$.

(b) There exists a morphisms $\varphi_{0}: X \rightarrow X_{0}$ such that $\operatorname{dp}\left(\varphi_{0}\right)=t$, for some $0 \leq t \leq$ $m-n$, and $f_{n} \ldots f_{1} \varphi_{0}+\mu \in \Re_{A}^{m+1}\left(X, X_{0}\right)$.

(c) If $\varphi_{0}$ is not an isomorphism then there exists a non-zero path of irreducible morphisms from $X$ to $X_{0}$ in $\bmod A$ of length at most $m-n$.

Proof. (a). By hypothesis there exists a sectional path

$$
\delta: X_{0} \stackrel{f_{1}}{\longrightarrow} X_{1} \stackrel{f_{2}}{\longrightarrow} \ldots \longrightarrow X_{n-1} \stackrel{f_{n}}{\longrightarrow} X_{n}
$$

with $\delta=f_{n} \ldots f_{1}$. By [?] we know that $\delta \in \Re_{A}^{n}\left(X_{0}, X_{n}\right) \backslash \Re_{A}^{n+1}\left(X_{0}, X_{n}\right)$. We also have that $f \delta=0$ then we get that $d_{l}(f) \leq n$. 
On the other hand, since $\tau_{A}^{-1} X_{n-2} \oplus X_{n}$ is the middle term of $\epsilon\left(X_{n+1}\right)$ by [?, Proposition 1.6] we get that $d_{l}(f) \geq n$. Hence, $d_{l}(f)=n$.

Now, since there is a morphism $\mu: X \rightarrow X_{n}$ with $X \in \Gamma$ such that $\operatorname{dp}(\mu)=m$ for some positive integer $m$ and $f \mu \in \Re_{A}^{m+2}\left(X, X_{n+1}\right)$, then, $d_{l}(f) \leq m$, that is, $n \leq m$.

(b). Since $\operatorname{dp}(\mu)=m$ and $f \mu \in \Re_{A}^{m+2}\left(X, X_{n+1}\right)$ by [?, Lemma 1.2] there is a morphism $\varphi_{n-1}: X \rightarrow X_{n-1}$ such that $\varphi_{n-1} \notin \Re_{A}^{m}\left(X, X_{n-1}\right), g_{n-1} \varphi_{n-1} \in \Re_{A}^{m+1}\left(X, \tau_{A}^{-1} X_{n-2}\right)$ and $f_{n} \varphi_{n-1}+\mu \in \Re_{A}^{m+1}\left(X, X_{n+1}\right)$. Then, $f_{n} \varphi_{n-1}=-\mu+\mu_{m+1}$ with $\mu_{m+1} \in \Re_{A}^{m+1}\left(X, X_{n}\right)$. Therefore, $\operatorname{dp}\left(f_{n} \varphi_{n-1}\right)=m$. Then, we infer that $\operatorname{dp}\left(\varphi_{n-1}\right)=r$ for some $n-1 \leq r<m$. In fact, assume that $r<n-1$. Note that in such a case $n>1$. If $\varphi_{n-1}$ is an isomorphism and since $\operatorname{dp}\left(f_{n} \varphi_{n-1}\right)=m$ then $\operatorname{dp}\left(f_{n}\right)=m$ but $m>1$, a contradiction to the fact that $f_{n}$ is an irreducible morphism. Then, $\varphi_{n-1}$ is not an isomorphism and $n \geq 2$.

With a similar argument as in the proof of Statement $(a)$ we have that $d_{l}\left(g_{n-1}\right)=n-1$, getting a contradiction to the fact that $\operatorname{since} \operatorname{dp}\left(\varphi_{n-1}\right)=r$ with $r<n-1$ and $g_{n-1} \varphi_{n-1} \in$ $\Re_{A}^{m+1}\left(X, \tau_{A}^{-1} X_{n-2}\right)$ then $d_{l}\left(g_{n-1}\right)<r<n-1$. Therefore, we prove that $\operatorname{dp}\left(\varphi_{n-1}\right)=r$ for some $n-1 \leq r \leq m-1$.

Now, since there is a morphism $\varphi_{n-1}: X \rightarrow X_{n-1}$ such that $\operatorname{dp}\left(\varphi_{n-1}\right)=r$ for some $n-1 \leq r \leq m-1$ and $g_{n-1} \varphi_{n-1} \in \Re_{A}^{m+1}\left(X, \tau_{A}^{-1} X_{n-2}\right)$ then by [?, Lemma 1.2] we have that there is a morphism $\varphi_{n-2}: X \rightarrow X_{n-2}$ such that $\varphi_{n-2} \notin \Re_{A}^{m-1}\left(X, X_{n-2}\right), g_{n-2} \varphi_{n-2} \in$ $\Re_{A}^{m}\left(X, \tau_{A}^{-1} X_{n-3}\right)$ and $f_{n-1} \varphi_{n-2}+\varphi_{n-1} \in \Re_{A}^{m}\left(X, X_{n-1}\right)$. With the same arguments as above we can show that $\operatorname{dp}\left(\varphi_{n-2}\right)=t$ for some $n-2 \leq t \leq m-2$. Moreover, $f_{n} f_{n-1} \varphi_{n-2}+\mu \in$ $\Re_{A}^{m+1}\left(X, X_{n+1}\right)$. In fact, since $f_{n-1} \varphi_{n-2}+\varphi_{n-1} \in \Re_{A}^{m}\left(X, X_{n}\right)$ then $f_{n} f_{n-1} \varphi_{n-2}+f_{n} \varphi_{n-1} \in$ $\Re_{A}^{m+1}\left(X, X_{n+1}\right)$ where $f_{n} \varphi_{n-1}=-\mu+\mu_{m+1}$ with $\mu_{m+1} \in \Re_{A}^{m+1}\left(X, X_{n+1}\right)$ getting that $f_{n} f_{n-1} \varphi_{n-2}+\mu \in \Re_{A}^{m+1}\left(X, X_{n+1}\right)$.

Iterating the same argument and applying successively [?, Lemma 1.2] to each possible morphism $\varphi_{i}: X \rightarrow X_{i}$ for $i=n-3, \ldots, 0$ we get that there is a morphism $\varphi_{0}: X \rightarrow X_{0}$ such that $\operatorname{dp}\left(\varphi_{0}\right)=t$, for some $0 \leq t \leq m-n$, and that $f_{n} \ldots f_{1} \varphi_{0}+\mu \in \Re_{A}^{m+1}\left(X, X_{n+1}\right)$. Observe that $\varphi_{0}$ can be an isomorphism.

(c). Since $\varphi_{0}$ is not an isomorphism then $m>n$ and therefore $0<t \leq m-n$. By [?, VI, Proposition 7.5] there exists a non-zero path of irreducible morphisms in $\bmod A$ of length at most $t$. Hence, we infer that there is a path of irreducible morphisms of length at most $m-n$, getting the result.

Lemma 3.7. Let $A$ be an artin algebra. Assume that there is a configuration of almost split sequences in $\bmod A$ as follows

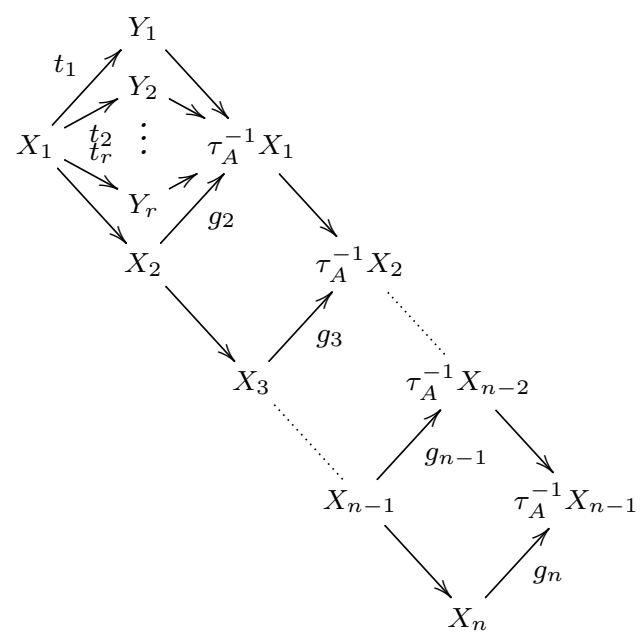


where $\alpha\left(X_{i}\right)=2$ for $2 \leq i \leq n-1$. Suppose there exists $1 \leq j \leq r$ such that $Y_{j}$ is projective and that the path $\delta: X_{1} \rightarrow \cdots \rightarrow X_{n-1} \rightarrow X_{n}$ is sectional. Consider $\delta_{i}: X_{1} \rightarrow \cdots \rightarrow X_{i}$ a subpath of $\delta$ and $g_{i}: X_{i} \rightarrow \tau_{A}^{-1} X_{i-1}$ irreducible morphisms. Then, $d_{l}\left(g_{i}\right)=\infty$ for $i \geq 2$. Moreover, the composition $g_{i} \delta_{i}$ behaves well for all $i \geq 2$.

Proof. Assume that $d_{l}\left(g_{k}\right)<\infty$, for some $2 \leq k \leq n-1$. By [?, Corollary 1.2] we know that $d_{l}\left(g_{2}\right)<\cdots<d_{l}\left(g_{n-1}\right)<d_{l}\left(g_{n}\right)$. Hence $d_{l}\left(g_{2}\right)<\infty$. Moreover, again by [?, Corollary 1.2] we get that $d_{l}\left(\left(t_{1}, \ldots, t_{r}\right)^{t}\right)<\infty$, but by our assumption there is an integer $1 \leq j \leq r$ such that $Y_{j}$ is projective getting a contradiction to [?, Lemma 1.2].

Finally, note that if $i \geq 2$ then the composition $g_{i} \delta_{i}$ behaves well, since $\delta_{i}$ is a sectional path and $d_{l}\left(g_{i}\right)=\infty$.

Lemma 3.8. Let $A$ be an artin algebra and $\Gamma$ be a component of $\Gamma_{A}$ with trivial valuation. Assume we have an exceptional wing $\mathcal{W}$ in $\Gamma$ as follows

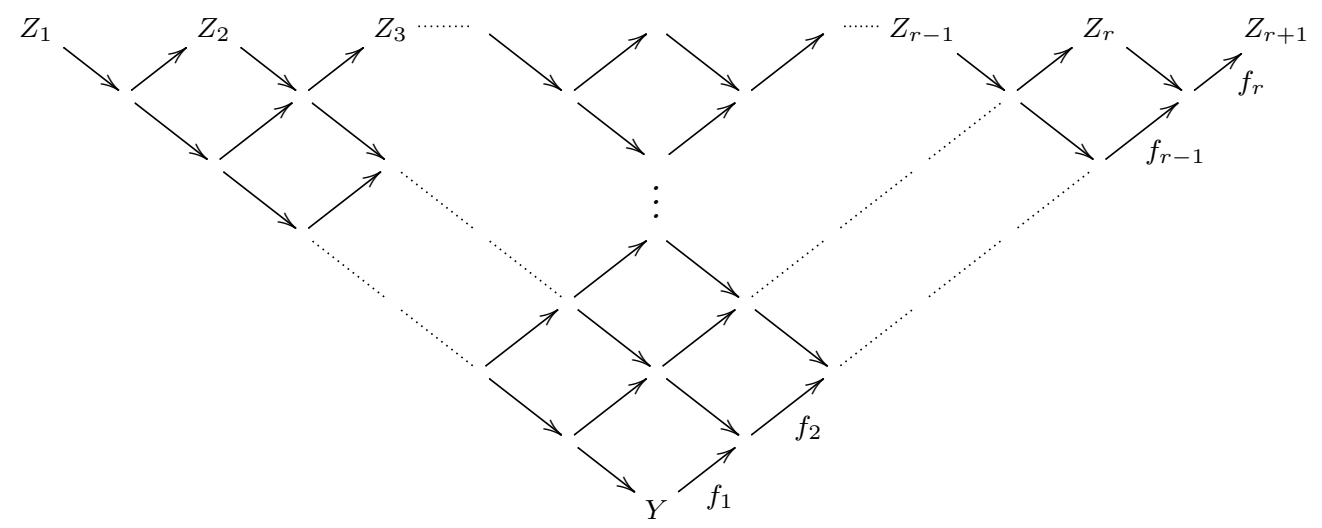

and that there exists $X \in \Gamma$ and a morphism $\mu: X \rightsquigarrow Y$ such that $\operatorname{dp}(\mu)=m$ with $m>r$. Moreover, assume that any path of irreducible morphisms from $X \rightsquigarrow Z_{i}$ in $\Gamma$ of length at most $m-r+2(i-1)$ is zero. Then, $\operatorname{dp}\left(f_{r} \ldots f_{1} \mu\right)=m+r$.

Proof. Assume that $f_{1} \mu \in \Re_{A}^{m+2}$. Since $d_{l}\left(f_{1}\right)=r$ then by Lemma ?? (b) there exists a morphism $\varphi_{0}: X \rightsquigarrow Z_{1}$ such that $\operatorname{dp}\left(\varphi_{0}\right)=t$ for some $0<t \leq m-r$.

Observe that since $m>r$ then $m-r>0$ and $X \not z Z_{1}$. Hence $\varphi_{0}$ is not an isomorphism. By Lemma ?? (c), we know that there exists a non-zero path of irreducible morphisms $\varphi^{\prime}: X \rightsquigarrow Z_{1}$ in $\bmod A$ of length at most $m-r$ with $\varphi^{\prime} \notin \Re_{A}^{m-r+1}\left(X, Z_{1}\right)$. We write the path $\varphi^{\prime}$ as follows

$$
\varphi^{\prime}: X \rightarrow Y_{1} \rightarrow Y_{2} \rightarrow \cdots \rightarrow Y_{j} \rightarrow Z_{1}
$$

On the other hand, if we consider $i=1$ then by hypothesis any path of irreducible morphisms in $\Gamma$ from $X \rightsquigarrow Z_{1}$ of length at most $m-r$ is zero. Therefore, any path $\gamma$ in $\Gamma$ going through the $A$-modules

$$
\gamma: X \rightarrow Y_{1} \rightarrow Y_{2} \rightarrow \cdots \rightarrow Y_{j} \rightarrow Z_{1}
$$

vanishes. Since we are considering $\Gamma$ a component of $\Gamma_{A}$ with trivial valuation then, by Lemma ?? we have that $\varphi^{\prime}=\delta \gamma+\mu$ with $\delta \in \operatorname{Aut}\left(Z_{1}\right)$ and $\mu \in \Re_{A}^{m-r+1}\left(X, Z_{1}\right)$. 
Hence, $\varphi^{\prime}=\mu$ with $\mu \in \Re_{A}^{m-r+1}\left(X, Z_{1}\right)$ getting a contradiction to the fact that $\varphi^{\prime} \notin$ $\Re_{A}^{m-r+1}\left(X, Z_{1}\right)$. Therefore, $\operatorname{dp}\left(f_{1} \mu\right)=m+1$.

Iterating this procedure over all the modules $Z_{i}$, for $2 \leq i \leq r$, we get that $\operatorname{dp}\left(f_{r} \ldots f_{1} \mu\right)$ $=m+r$.

Now, applying Proposition ?? and the above lemmas we get the announced result concerning compositions of irreducible morphisms of the borders of an exceptional wing.

Proposition 3.9. Let $A$ be an artin algebra and $\Gamma$ a component of $\Gamma_{A}$ with trivial valuation. Assume we have an exceptional wing $\mathcal{W}$ in $\Gamma$ containing a configuration of $n$ almost split sequences with exactly three indecomposable middle terms as follows:

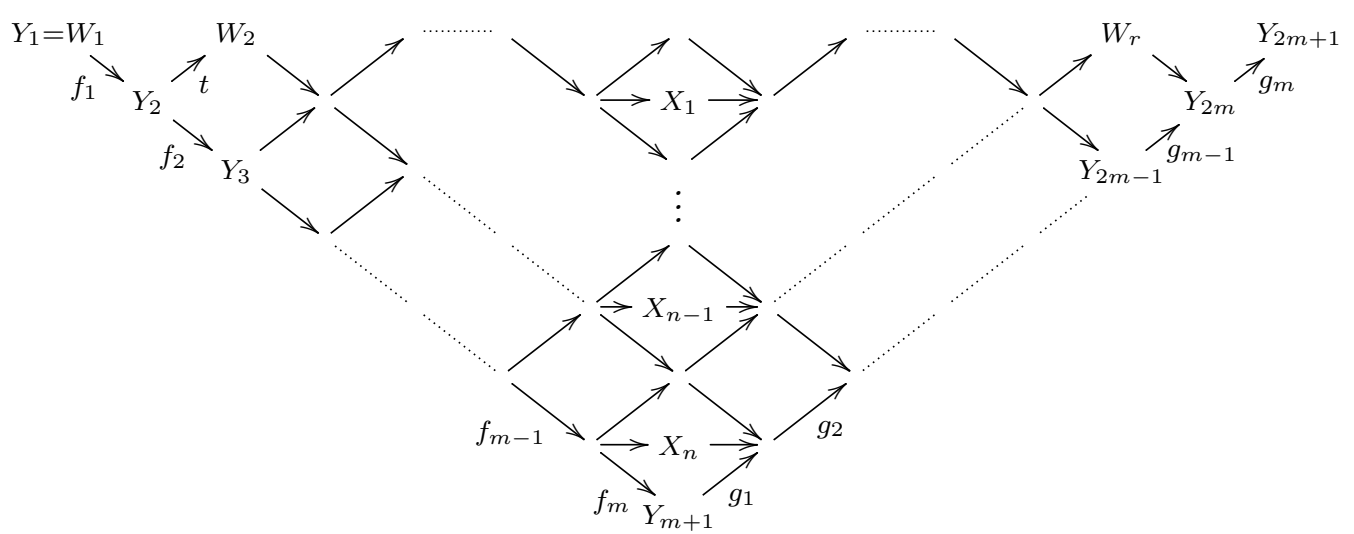

Then,

(a) The composition of irreducible morphisms in $\bmod A$ between the indecomposable $A$ modules of the borders of $\mathcal{W}$ behaves well.

(b) Any composition of irreducible morphisms in $\mathcal{W}$ from $W_{1}$ to $W_{j}, 2 \leq j \leq r$ is zero.

Proof. Let $\mathcal{W}$ be an exceptional wing in $\Gamma$. Without loss of generality, it is enough to consider an exceptional wing as in the statement with $X_{i} \neq 0$ for $i=n$.

(a). First, if we consider a path involving the modules $Y_{i}$ for $i=1, \ldots, m+1$ since any such a path is sectional we get the result by [?].

Now, by Lemma ?? we know that $\operatorname{dp}\left(g_{1} f_{m} \ldots f_{1}\right)=m+1$ since $f_{m} \ldots f_{1}$ is a sectional path and $d_{l}\left(g_{1}\right)=\infty$.

Next, we proceed as follows. If $X_{n-1}$ is projective module then $d_{l}\left(g_{2}\right)=\infty$. Hence $\operatorname{dp}\left(g_{2} g_{1} f_{m} \ldots f_{1}\right)=m+2$ since $\operatorname{dp}\left(g_{1} f_{m} \ldots f_{1}\right)=m+1$. Otherwise, $X_{n-1}=0$ and by Lemma ?? (a) we have that $d_{l}\left(g_{2}\right)=m-1$.

Assume that $g_{2} g_{1} f_{m} \ldots f_{1} \in \Re_{A}^{m+3}\left(W_{1}, Y_{m+2}\right)$. Then, by Lemma ?? (c) there exists a non-zero path in $\bmod A$ of length at most 2 from $W_{1} \rightsquigarrow W_{2}$. Note that any path in $\Gamma$ from $W_{1}$ to $W_{2}$ of length 2 is zero. In fact, observe that the only path of length two in $\Gamma$ from $W_{1}$ to $W_{2}$ is the path $W_{1}=Y_{1} \stackrel{f_{1}}{\longrightarrow} Y_{2} \stackrel{t}{\longrightarrow} W_{2}$ whose irreducible morphisms belong to an almost split sequence with indecomposable middle term. Hence, $t f_{1}=0$. Since the arrows of $\Gamma$ have trivial valuation then by Lemma ?? any other path of irreducible morphisms of length two between the same modules, let say, $W_{1}=Y_{1} \stackrel{h_{1}}{\longrightarrow} Y_{2} \stackrel{h_{2}}{\longrightarrow} W_{2}$, is such that $h_{2} h_{1}=\delta t f_{1}+\mu$ with $\delta \in \operatorname{Aut}\left(W_{2}\right)$ and $\mu \in \Re_{A}^{3}\left(W_{1}, W_{2}\right)$. Then $h_{2} h_{1} \in \Re_{A}^{3}\left(W_{1}, W_{2}\right)$. If 
$h_{2} h_{1} \neq 0$ then we get a contradiction to Lemma ?? $(c)$. Therefore, we prove that we can not have a non-zero path of irreducible morphisms between $W_{1}$ and $W_{2}$ of length at most two. Then, by Lemma ?? we get that $\operatorname{dp}\left(g_{2} g_{1} f_{m} \ldots f_{1}\right)=m+2$.

Analyzing the composition of each irreducible morphism $g_{i}$ with $3 \leq i \leq n$ as above we get that $\operatorname{dp}\left(g_{n} \ldots g_{1} f_{m} \ldots f_{1}\right)=m+n$. Finally, applying Lemma ??, we get the result.

Furthermore, any composition of the form $g_{s} \ldots g_{1} f_{m} \ldots f_{r}$ with $1 \leq s \leq m$ and $1 \leq r \leq m$ also behaves well.

(b). It is an immediate consequence of the fact that all such paths may go through the almost split sequence starting at $W_{1}$ which has exactly one indecomposable middle term.

Proposition 3.10. Let $A$ be an artin algebra and $\Gamma$ a component of $\Gamma_{A}$ with trivial valuation. Assume we have an exceptional wing $\mathcal{W}$ in $\Gamma$ containing a configuration of $n$ almost split sequences with exactly three indecomposable middle terms as follows:

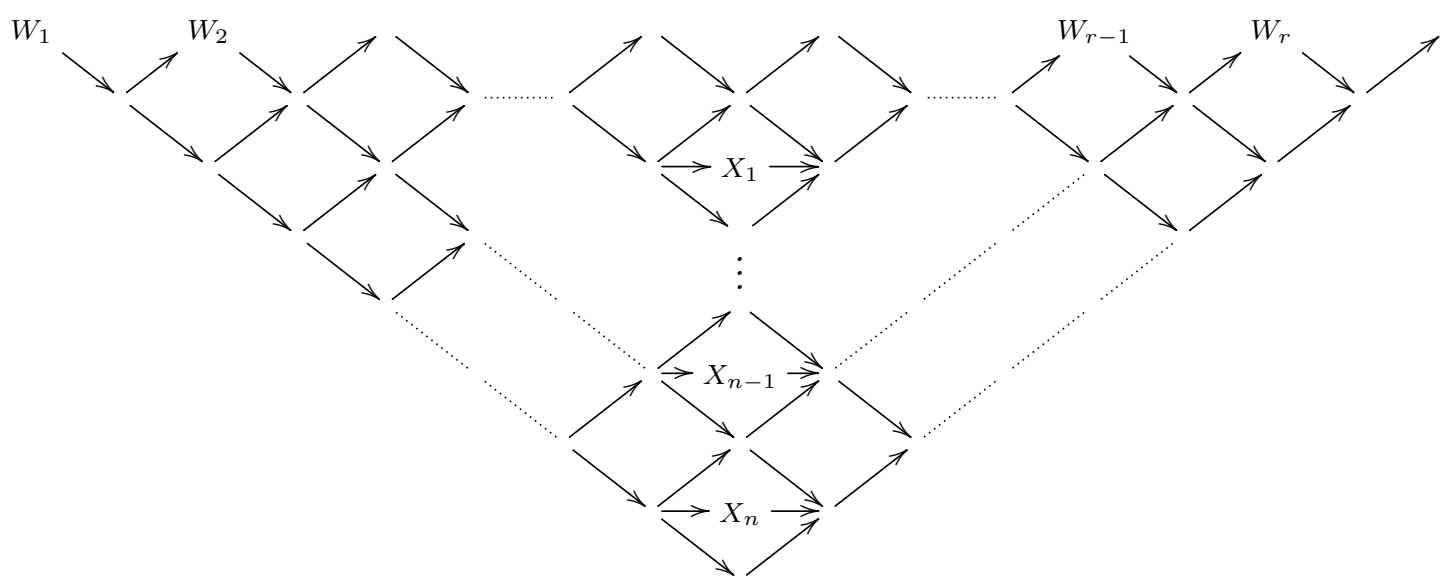

Then,

(a) The composition of irreducible morphisms in $\bmod A$ between the indecomposable $A$ modules of the borders of $\mathcal{W}$ behaves well.

(b) Any composition of irreducible morphisms in $\mathcal{W}$ from $W_{1}$ to $W_{j}, 2 \leq j \leq r$ is zero.

Proof. Similar to the proof of Proposition ??.

Proposition 3.11. Let $A$ be an artin algebra and $\Gamma \subset \Gamma_{A}$ a smooth quasi-tube with only one almost split sequence with three indecomposable middle terms. Then the following conditions hold.

(a) The composition of $r \geq 1$ cycles in $\Gamma(\bmod A)$ from a projective-injective indecomposable module $P$ with $\mathrm{sql}(P)=\operatorname{rank}\left(\Gamma^{s}\right)$ (respectively, immediate predecessor or successor of $P$ ) behaves well.

(b) If $\operatorname{sql}(P)<\operatorname{rank}\left(\Gamma^{s}\right)$ then the composition of $r \geq 1$ cycles in $\Gamma(\bmod A)$ from a projective-injective indecomposable module $P$ (respectively, immediate predecessor or successor of $P$ ) is zero. 
Proof. Let $A$ be an artin algebra, $\Gamma$ be a smooth quasi-tube in $\Gamma_{A}$, and $P$ be a projectiveinjective module in $\Gamma$ (respectively, immediate predecessor or successor of $P$ ). Observe first that, it follows from [?, Lemma 4.9] (see also [?, Lemmas 2.5-2.8 and their duals]) that $\operatorname{sql}(P) \leq \operatorname{rank}\left(\Gamma^{s}\right)$.

(a). We illustrate the situation of this statement with the following diagram

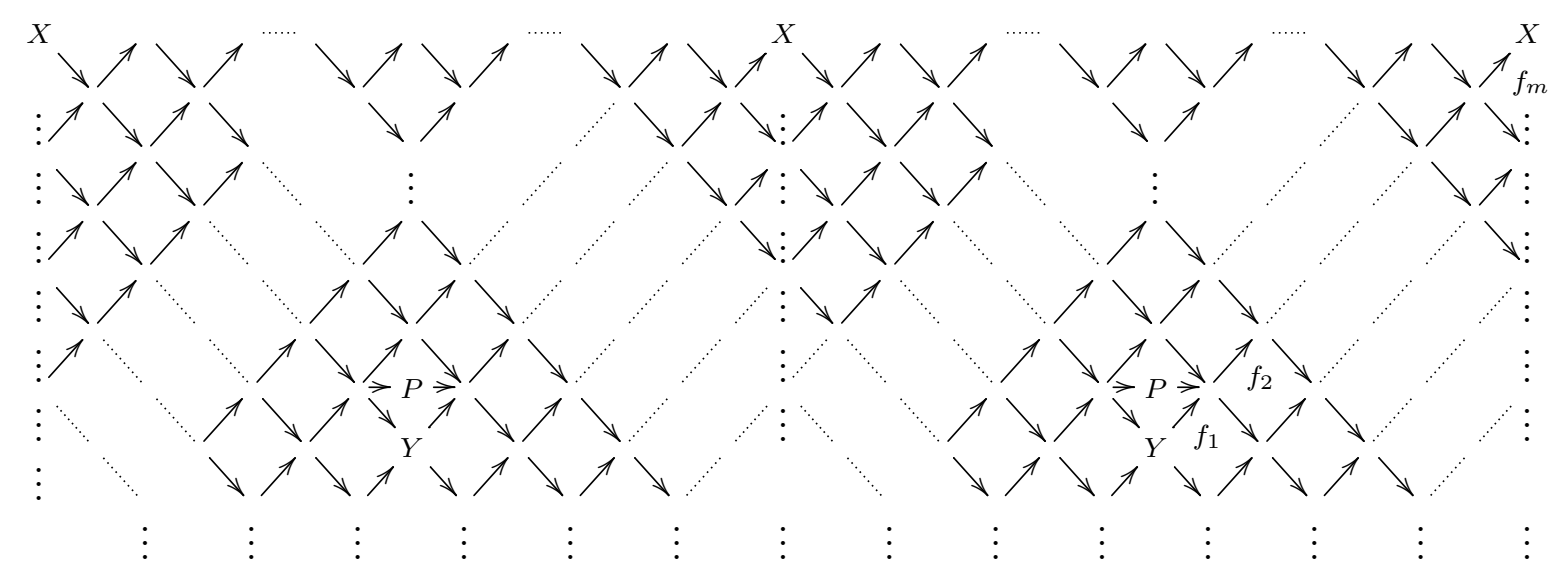

By Proposition ?? we know that the composition of the morphisms in the borders $\delta_{1}$ starting at $X$ and ending at $Y$ and $\delta_{2}$ starting at $Y$ and ending at $X$ of the exceptional wing, behaves well. Moreover, $f_{1} \delta_{1} \delta_{2} \delta_{1}$ also behaves well since $d_{l}\left(f_{1}\right)=\infty$ and the irreducible morphisms of the border $\delta_{1}$ have infinite left degree. By Lemma ??, we know that $f_{m} \ldots f_{1} \delta_{1} \delta_{2} \delta_{1}=\delta_{2} \delta_{1} \delta_{2} \delta_{1}$ behaves well.

Repeating this argument we get the result for $\Gamma$. Moreover, by Lemma ?? we get the result for $\bmod A$.

(b). Let $P$ be a projective-injective module, $\operatorname{sql}(P)<\operatorname{rank}\left(\Gamma^{s}\right)$ and

$$
X_{m} \rightarrow X_{m-1} \rightarrow \cdots \rightarrow X_{1} \rightarrow X_{0}=P
$$

be the unique sectional path in $\Gamma$ with $X_{m}$ lying on the mouth of $\Gamma^{s}$. Then $m=\operatorname{sql}(P)$. Observe that the smooth quasi-tube $\Gamma$ is a coherent component of $\Gamma_{A}$ (??). Since $\Gamma$ is also a cyclic component of $\Gamma_{A}$, applying [?, Theorem A] (see also [?, Theorem 2.3]), we infer that $\Gamma$, considered as a translation quiver, can be obtained from a stable tube by an iterated application of admissible operations of type $(\operatorname{ad} 1),(\operatorname{ad} 2),\left(\operatorname{ad} 1^{*}\right)$ and $\left(\operatorname{ad} 2^{*}\right)$, described in Section ??. Moreover, by our assumption on the number of almost split sequences with three middle terms, we can apply only one admissible operation of type $($ ad 2$)$ or $\left(\operatorname{ad} 2^{*}\right)$. Then it follows that $\operatorname{Hom}_{A}\left(P, X_{i}\right)=0$ and $\operatorname{Hom}_{A}\left(P, Y_{j}\right)=0$, where

$$
X_{m} \rightarrow X_{m-1} \rightarrow \cdots \rightarrow X_{1} \rightarrow Y_{1} \rightarrow Y_{2} \rightarrow \cdots
$$

is the unique infinite sectional path in $\Gamma$ consisting of arrows pointing to infinity and $1 \leq i \leq m, j \geq 1$. Therefore, $\operatorname{Hom}_{A}\left(P, X_{0}\right)=\operatorname{Hom}_{A}(P, P)=0$ and then the composition of $r \geq 1$ cycles in $\Gamma(\bmod A)$ from $P$ (respectively, from immediate predecessor or successor of $P$ ) is zero.

Proposition 3.12. Let $A$ be an artin algebra and $\Gamma$ a smooth quasi-tube in $\Gamma_{A}$. Assume we have an exceptional wing $\mathcal{W}$ in $\Gamma$ containing a configuration of $n$ almost split sequences with exactly three indecomposable middle terms as on the figures in Definition ??. Then the following conditions hold. 
(a) The composition of $r \geq 1$ cycles in $\Gamma(\bmod A)$ from a projective-injective indecomposable module $X_{n}$ with $\operatorname{sql}\left(X_{n}\right)=\operatorname{rank}\left(\Gamma^{s}\right)$ (respectively, immediate predecessor or successor of $X_{n}$ ) behaves well.

(b) If $\operatorname{sql}\left(X_{n}\right)<\operatorname{rank}\left(\Gamma^{s}\right)$ then the composition of $r \geq 1$ cycles in $\Gamma(\bmod A)$ from a projective-injective indecomposable module $X_{n}$ (respectively, immediate predecessor or successor of $X_{n}$ ) is zero.

Proof. Similar to the proof of Proposition ?? using additionally induction on the number of projective-injective modules.

The next result shall be useful for further purposes.

Lemma 3.13. Let $A$ be a selfinjective artin algebra and $\Gamma \subset \Gamma_{A}$ be a quasi-tube. Assume we have in $\Gamma$ a zero path of irreducible morphisms $X_{1} \rightarrow X_{2} \rightarrow \cdots \rightarrow X_{n} \rightarrow X_{n+1}$. Then, any longest path in $\Gamma$ from $X_{1} \rightsquigarrow X_{n+1}$ vanishes.

Proof. Let $A$ be a selfinjective artin algebra and $\Gamma$ be a quasi-tube in $\Gamma_{A}$. It follows from Proposition ?? that the stable part $\Gamma^{s}$ of $\Gamma$ is a stable tube. Moreover, observe that the quasi-tube $\Gamma$ is a coherent component of $\Gamma_{A}$, that is, the following two conditions are satisfied:

(C1) For each projective module $P$ in $\Gamma$ there is an infinite sectional path $P=U_{1} \rightarrow$ $U_{2} \rightarrow U_{3} \rightarrow \cdots$ starting at $P$

(C2) For each injective module $I$ in $\Gamma$ there is an infinite sectional path $\cdots \rightarrow V_{3} \rightarrow V_{2} \rightarrow$ $V_{1}=I$ ending at $I$.

Since $\Gamma$ is also a cyclic component of $\Gamma_{A}$, applying [?, Theorem A] (see also [?, Theorem $2.3]$ ), we infer that $\Gamma$, considered as a translation quiver, can be obtained from a stable tube by an iterated application of admissible operations of type $(\operatorname{ad} 1),(\operatorname{ad} 2),\left(\operatorname{ad} 1^{*}\right)$ and $\left(\right.$ ad $\left.2^{*}\right)$. Since the projectives and injectives vertices in $\Gamma$ coincide, the projective-injective vertices in $\Gamma$ are created as follows:

- for each operation $(\operatorname{ad} 1)$ with pivot $X_{0}$ and $t=0$, the operation $\left(\operatorname{ad} 1^{*}\right)$ with pivot at $X_{0}^{\prime}$ and $t=0$ is applied;

- for each operation $\left(\operatorname{ad} 1^{*}\right)$ with pivot $X_{0}$ and $t=0$, the operation (ad 1) with pivot at $X_{0}^{\prime}$ and $t=0$ is applied;

- for each operation $(\operatorname{ad} 1)$ with pivot $X_{0}$ and $t \geq 1$, the operation $\left(\operatorname{ad} 2^{*}\right)$ with pivot at $Z_{01}$ is applied;

- for each operation $\left(\operatorname{ad} 1^{*}\right)$ with pivot $X_{0}$ and $t \geq 1$, the operation $(\operatorname{ad} 2)$ with pivot at $Z_{01}$ is applied.

Now, let $\alpha: X_{1} \rightarrow X_{2} \rightarrow \cdots \rightarrow X_{n} \rightarrow X_{n+1}$ be a zero path of irreducible morphisms in $\Gamma$ and $i$ be the largest index such that a subpath $\beta: X_{1} \rightarrow X_{2} \rightarrow \cdots \rightarrow X_{i}$ of $\alpha$ is non-zero. Moreover, let $Z_{p} \rightarrow Z_{p-1} \rightarrow \cdots \rightarrow Z_{1} \rightarrow X_{i}$ be the unique maximal sectional path in $\Gamma$ starting at $Z_{p}$ and formed by arrows pointing to the infinity. Then $Z_{p}$ lies on the mouth of $\Gamma^{s}$. Then, it follows from the definition of admissible operations of types $(\operatorname{ad} 1),(\operatorname{ad} 2)$, $\left(\right.$ ad $\left.1^{*}\right),\left(\right.$ ad $\left.2^{*}\right)$ that, if $X_{1} \rightarrow \cdots \rightarrow Y$ is a non-zero path of irreducible morphisms in $\Gamma$ then $Y$ lies in the infinite rectangle $\mathscr{S}\left(X_{1}, Z_{p}\right)$ consisting of the vertices bounded by: 
- the infinite sectional path in $\Gamma$ starting at $X_{1}$ and formed by arrows pointing to the infinity;

- the finite sectional path in $\Gamma$ starting at $X_{1}$ and formed by arrows pointing to the mouth;

- the infinite sectional path $Z_{p} \rightarrow \cdots \rightarrow Z_{1} \rightarrow X_{i} \rightarrow \cdots$ in $\Gamma$ starting at $Z_{p}$ and formed by arrows pointing to the infinity.

Therefore, any longest path in $\Gamma$ from $X_{1}$ to $X_{n+1}$ vanishes.

Our next result shows that if $A$ is a selfinjective artin algebra and $\Gamma$ an infinite component of $\Gamma_{A}$ without special configurations of modules and containing an oriented cycle then the composition of irreducible morphisms $f_{n} \ldots f_{1} \in \Re_{A}^{n+1}\left(X_{1}, X_{n+1}\right)$ if and only if $f_{n} \ldots f_{1} \in \Re_{A}^{\infty}\left(X_{1}, X_{n+1}\right)$. To achive to such a result we start proving the following lemma.

Lemma 3.14. Let $A$ be a selfinjective artin algebra and $\Gamma$ be a quasi-tube in $\Gamma_{A}$ with at least two projective-injective modules and such that all projective-injectives belong to exactly two exceptional wings $\mathcal{W}$ and $\mathcal{W}^{\prime}$ in $\Gamma$. Let $\alpha: X \rightarrow \cdots \rightarrow Y, \beta: Y \rightarrow \cdots \rightarrow Z$ be the borders of $\mathcal{W}$ and $\gamma: U \rightarrow \cdots \rightarrow V, \delta: V \rightarrow \cdots \rightarrow W$ be the borders of $\mathcal{W}^{\prime}$. Then the following conditions hold.

(a) If $Z=U$ (respectively, $W=X$ ) then the composition of irreducible morphisms from $X$ to $W$ (respectively, from $U$ to $Z$ ) behaves well.

(b) If $Z \neq U$ (respectively, $W \neq X$ ) then any composition of irreducible morphisms from $X$ to $W$ (respectively, from $U$ to $Z$ ) is zero.

Proof. Observe first that, it follows from [?, Lemma 4.9] (see also [?, Lemmas 2.5-2.8]) that there is in $\Gamma$ the infinite rectangle $\mathscr{S}(Y, Z)$ consisting of the vertices bounded by:

- the infinite sectional path in $\Gamma$ starting at $Y$ and formed by arrows pointing to the infinity;

- the finite sectional path $\beta: Y \rightarrow \cdots \rightarrow Z$ in $\Gamma$;

- the infinite sectional path in $\Gamma$ starting at $Z$ and formed by arrows pointing to the infinity.

Moreover, all meshes in $\mathscr{S}(Y, Z)$ are with exactly two middle terms and for any $T$ from $\mathscr{S}(Y, Z)$ we have $\operatorname{Hom}_{A}(X, T) \neq 0$.

Let $Z=U$. By Proposition ?? we know that the composition of irreducible morphisms of the borders of the exceptional wings behaves well. Let $\sigma$ be the sectional path in $\Gamma$ from infinity to $V$ and $\varrho$ be the sectional path in $\Gamma$ from $Y$ to infinity. Then $\sigma$ intersects $\varrho$ and denote by $N$ their common module. Note that every composition of irreducible morphisms from $Y$ to $V$ in the rectangle $\mathscr{S}(Y, Z, V, N)$ is equal and non-zero. Therefore, the composition of irreducible morphisms from $X$ to $W$ behaves well.

If $Z \neq U$, then for the infinite sectional path $\tau_{A}^{-} Z=M_{1} \rightarrow M_{2} \rightarrow \cdots$ formed by arrows pointing to the infinity we have $\operatorname{Hom}_{A}\left(X, M_{i}\right)=0$ where $i \geq 1$ and $Z$ is the starting vertex of a mesh with exactly one middle term. Hence we get (b). 
Theorem 3.15. Let $A$ be a selfinjective artin algebra and $\Gamma$ a quasi-tube of $\Gamma_{A}$ without special configurations of modules. Let

$$
X_{1} \stackrel{f_{1}}{\longrightarrow} X_{2} \stackrel{f_{2}}{\longrightarrow} \cdots \stackrel{f_{n-1}}{\longrightarrow} X_{n} \stackrel{f_{n}}{\longrightarrow} X_{n+1}
$$

be a path of irreducible morphisms with $X_{i} \in \Gamma$ for $i=1, \ldots, n+1$. Then, $f_{n} \ldots f_{1} \in$ $\Re_{A}^{n+1}\left(X_{1}, X_{n+1}\right)$ if and only if $f_{n} \ldots f_{1} \in \Re_{A}^{\infty}\left(X_{1}, X_{n+1}\right)$.

Proof. We only prove that, if $f_{n} \ldots f_{1} \in \Re_{A}^{n+1}\left(X_{1}, X_{n+1}\right)$ then $f_{n} \ldots f_{1} \in \Re_{A}^{\infty}\left(X_{1}, X_{n+1}\right)$ since the other implication is clear.

To analyze the composition of irreducible morphisms in $\Gamma$ we will start with the ones near the mouth of $\Gamma$. It is enough to study that all non-zero compositions behaves well.

We will do induction on the number $n+m$ with $n, m \geq 1$, where $n$ is the number of exceptional wings in $\Gamma$ and $m$ is the number of projective-injective vertices from almost split sequences with exactly two middle terms in $\Gamma$. We would like to note that on the figures below we present the first exceptional wing from Definition ?? but for the second exceptional wing containing the meshes with exactly three middle terms, the proof is the same. Let $n+m=2$, we have three cases.

(a). If $n=2$ then by Lemma ?? we get the result.

(b). Let $n=1$ and $m=1$. Let $P$ be a projective-injective module in $\Gamma$ belonging to a mesh with exactly two middle terms. Consider an exceptional wing $\mathcal{W}$ with the borders $\varphi_{1}$ starting at $X$ and $\varphi_{2}$ ending in $Y$. Then, the only non-zero composition of irreducible morphisms from $X$ to $Z$ is $g_{s} \ldots g_{1} \varphi_{2} \varphi_{1}$, where $g_{s} \ldots g_{1}$ belong to the unique infinite sectional path in $\Gamma$ starting at $Y$ and passing through $Z$ (formed by arrows pointing to the infinity), and $\varphi_{2} \varphi_{1}$ is the composition of the borders of the wing $\mathcal{W}$. We illustrate the situation with the following diagram:

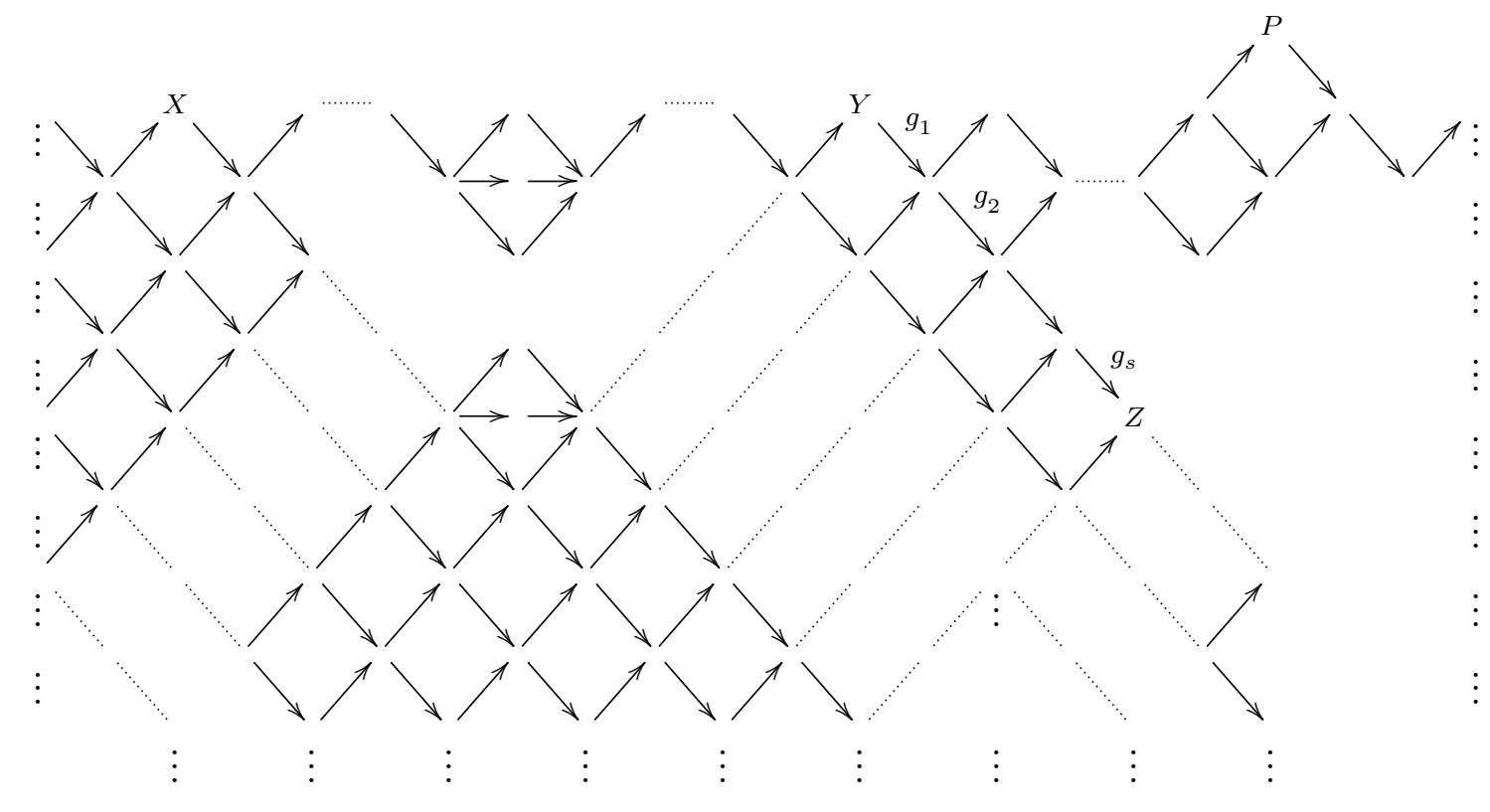

In fact, by Proposition ?? or Proposition ?? the composition $\varphi_{2} \varphi_{1}$ of the borders of the exceptional wing $\mathcal{W}$ behaves well. Now, since the left degree of the morphisms $g_{1}, \ldots, g_{s}$ 
are infinite then the composition $g_{s} \ldots g_{1} \varphi_{2} \varphi_{1}$ behaves well. Now, consider the situation illustrated with the following diagram:

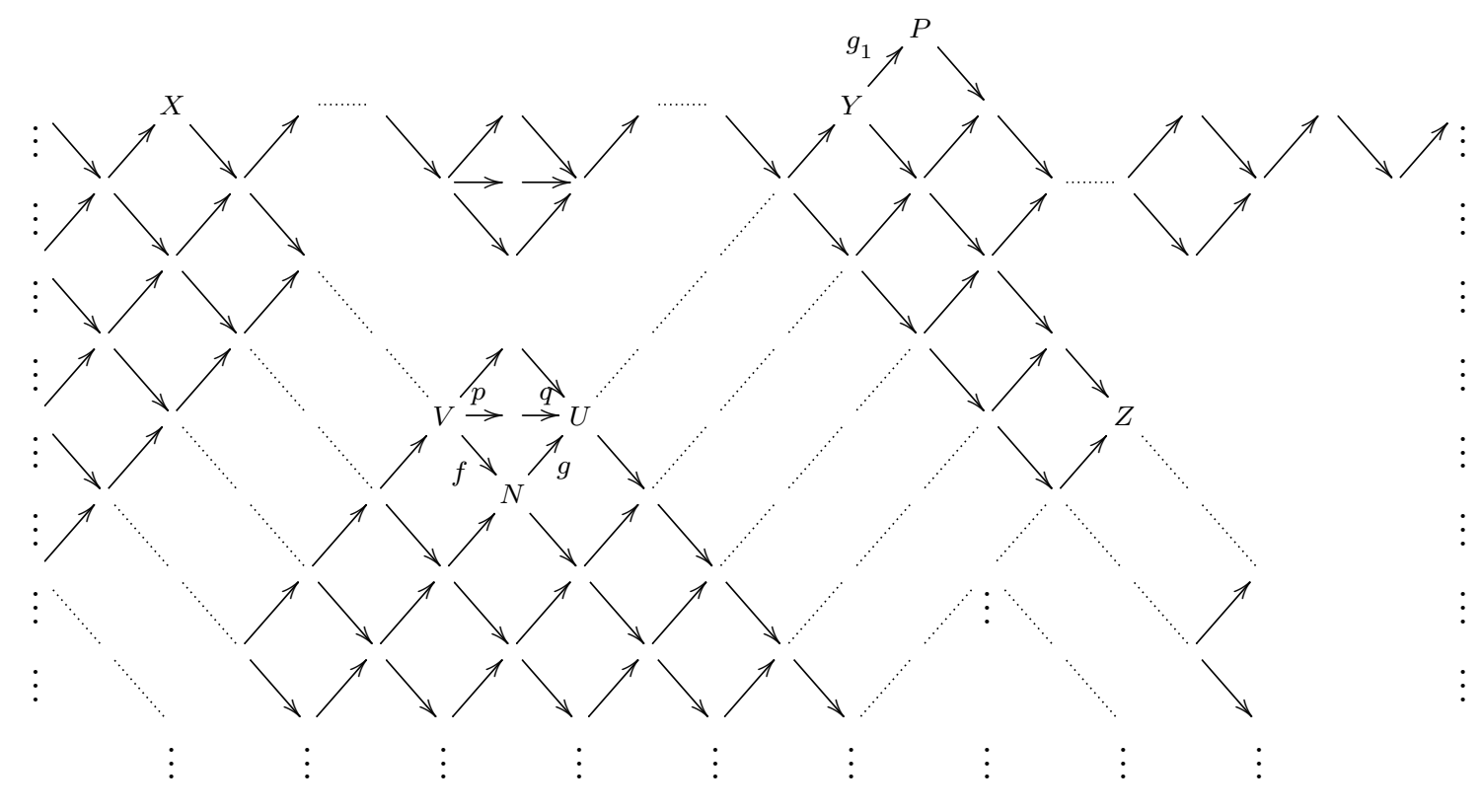

Denote by $\varphi$ the unique sectional path from $X$ to $V$, by $\psi$ the unique sectional path from $U$ to $Y$, and by $\eta$ the unique sectional path from $P$ to $\tau_{A}^{-} Z$. Note that the compositions $\eta g_{1} \psi q p \varphi$ and $\eta g_{1} \psi g f \varphi$ behaves well, since by [?] $d_{l}\left(g_{1}\right)=\infty$ and by [?] the irreducible morphisms in $\eta$ have infinite left degree. Therefore, the only non-zero composition of irreducible morphisms from $X$ to $\tau_{A}^{-} Z$ passing through $P$ behaves well.

$(c)$. Let $m=2$ and $X, Y$ be the projective-injective modules in $\Gamma$. In this case, if $\operatorname{Hom}_{A}(X, Z) \neq 0$ (respectively, $\operatorname{Hom}_{A}(Y, Z) \neq 0$ ) then $Z$ belongs to the unique infinite sectional path starting at $X$ (respectively, at $Y$ ). Moreover, it follows by Lemma ?? that any path from $X$ to $Y$ is zero. The non-zero paths are the ones which involves almost split sequences not going through (modulo mesh) the almost split sequences with only one indecomposable middle term. In fact, this follows because one can write such a composition as a chain of irreducible morphisms of a coray followed by a chain of irreducible morphisms in a ray. By [?] the right degree of the ones in the coray are infinite and the left degree of the ones in the ray are infinite.

Assume that for $n+m-1$ the result is true. We want to prove our theorem for $n+m$. We have two cases:

Case 1. We fix a configuration of almost split sequences of an exceptional wing $\mathcal{W}$ as follows: 


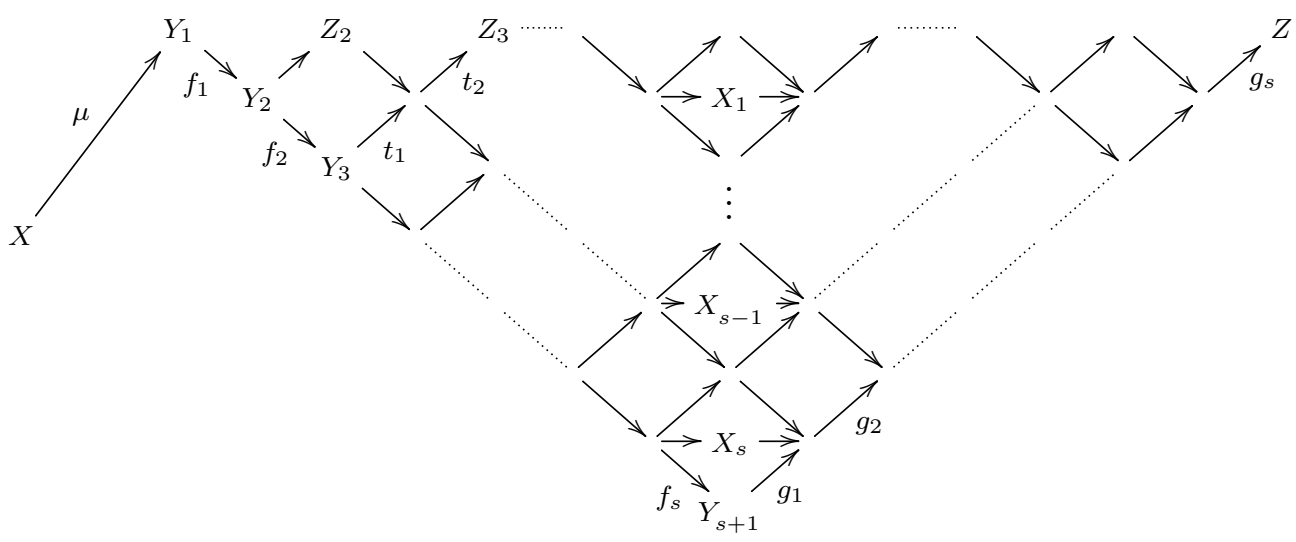

Since the left degrees of $f_{1}, \ldots, f_{s}, g_{1}$ are infinite then $g_{1} f_{s} \ldots f_{1} \mu$ behaves well. Now we proceed as in the proof of Proposition ??, that is, if $X_{s-1}$ is projective-injective then $d_{l}\left(g_{2}\right)=\infty$ and hence $g_{2} g_{1} f_{s} \ldots f_{1} \mu$ behaves well. Otherwise, if $X_{s-1}=0$ then by Lemma ?? we know that any path in $\Gamma$ from $Y_{1}$ to $Z_{3}$, say $\varphi_{1}: Y_{1} \rightsquigarrow Z_{3}$, is zero. Therefore, clearly, any longest path as $t_{2} t_{1} f_{2} f_{1} \mu$ is also zero. Iterating this procedure, we get that the composition $g_{s} \ldots g_{2} g_{1} f_{s} \ldots f_{1} \mu$ behaves well. On the other hand, if $\mu: X \rightarrow Y_{i}$ for $2 \leq i \leq s$ then by inductive hypothesis we have that $\mu$ behaves well. Then, we have to consider the last configuration next to $\mathcal{W}$. We have two cases to consider.

Case 1-1. Consider the situation illustrated with the following diagram:

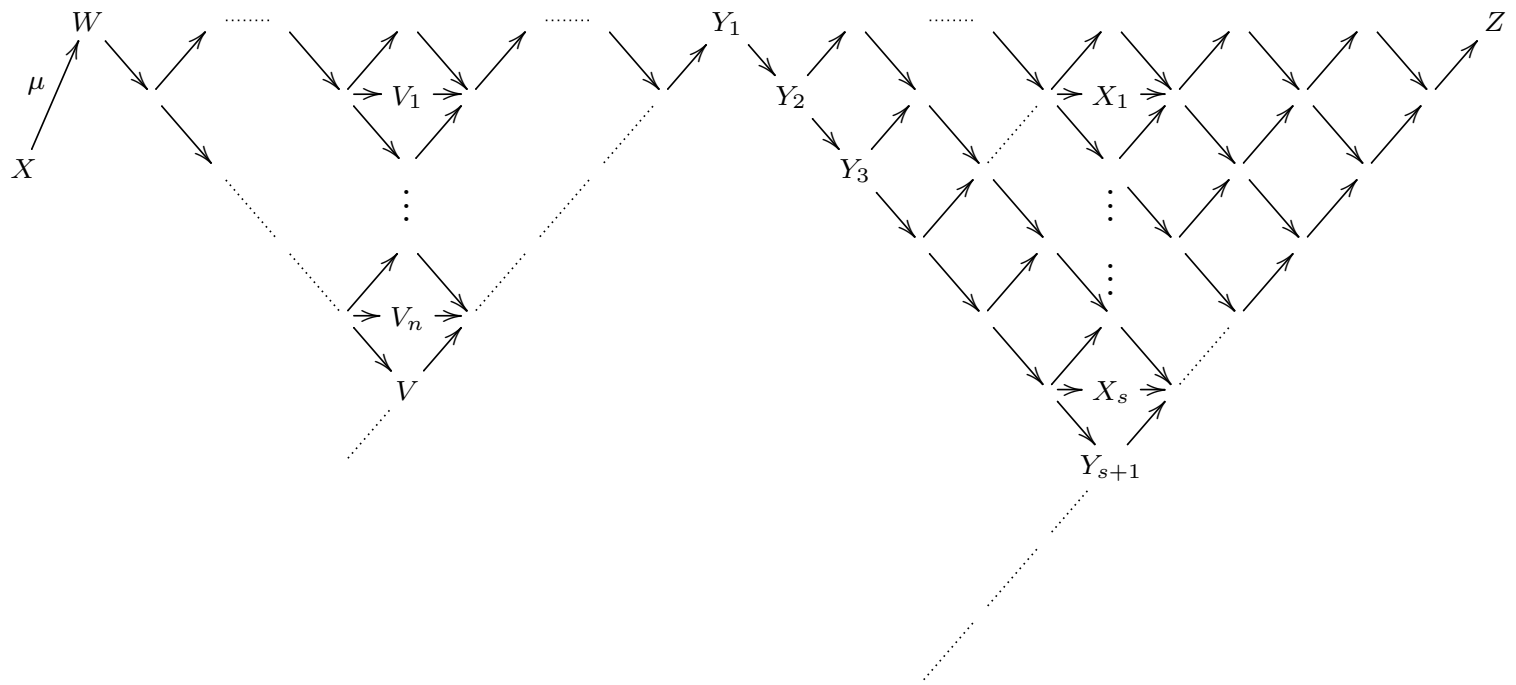

Then it follows from the inductive hypothesis, Lemma ?? and its proof that the composition $\lambda_{2} \lambda_{1} \sigma_{2} \sigma_{1} \mu$ behaves well, where $\sigma_{1}: W \rightarrow \cdots \rightarrow V, \sigma_{2}: V \rightarrow \cdots \rightarrow Y_{1}$, $\lambda_{1}: Y_{1} \rightarrow \cdots \rightarrow Y_{s+1}, \lambda_{2}: Y_{s+1} \rightarrow \cdots \rightarrow Z$ are the borders of the above exceptional wings, and $\mathscr{S}\left(Y_{1}, Y_{s+1}\right)$ is the infinite rectangle consisting of the vertices bounded by:

- the infinite sectional path in $\Gamma$ ending at $Y_{1}$ and formed by arrows pointing to the mouth;

- the finite sectional path $Y_{1} \rightarrow \cdots \rightarrow Y_{s+1}$ in $\Gamma$; 
- the infinite sectional path in $\Gamma$ ending at $Y_{s+1}$ and formed by arrows pointing to the mouth.

Moreover, all meshes in $\mathscr{S}\left(Y_{1}, Y_{s+1}\right)$ are with exactly two middle terms and for any $U$ from $\mathscr{S}\left(Y_{1}, Y_{s+1}\right)$ we have $\operatorname{Hom}_{A}(U, Z) \neq 0$.

Case 1-2. Consider the situation illustrated with the following diagram:

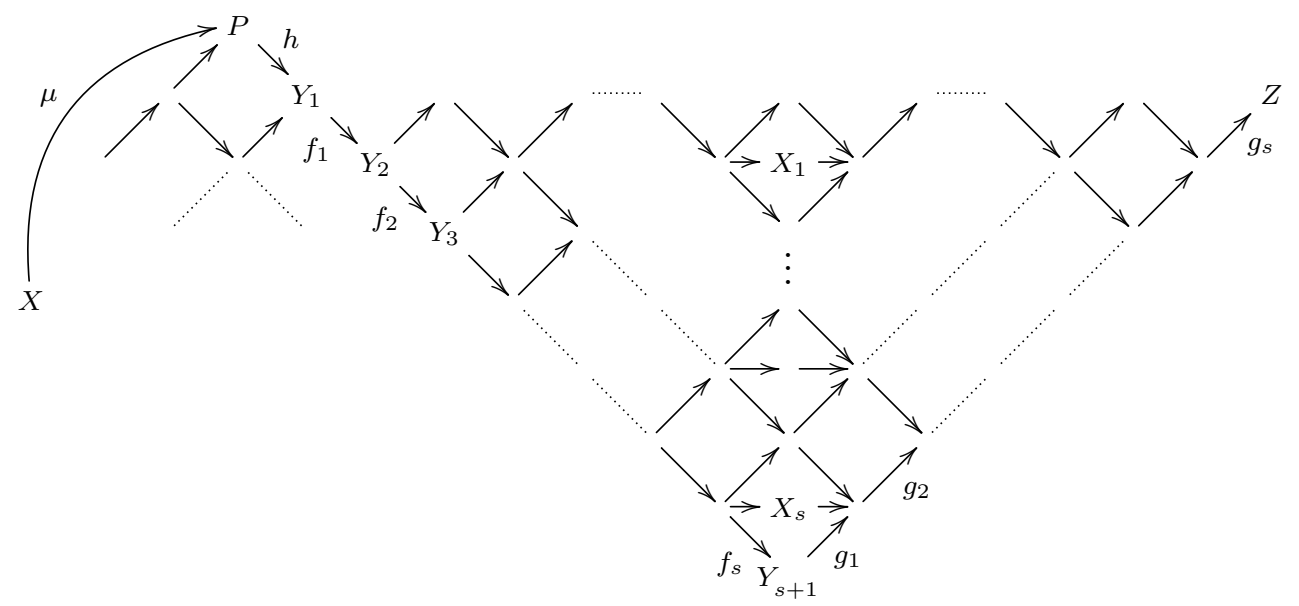

with the exceptional wing $\mathcal{W}$ and projective-injective module $P$ in $\Gamma$ belonging to a mesh with exactly two middle terms. By the previous considerations it is enough to consider the composition $g_{s} \ldots g_{1} f_{s} \ldots f_{1} h \mu$. By the inductive hypothesis $\mu: X \rightsquigarrow P$ behaves well. Since the left degree of irreducible morphisms $h, f_{1}, \ldots, f_{s}$ are infinite then the composition $f_{s} \ldots f_{1} h \mu$ behaves well. Finally, also $g_{s} \ldots g_{1} f_{s} \ldots f_{1} h \mu$ behaves well.

Case 2. Assume we have the following situation:

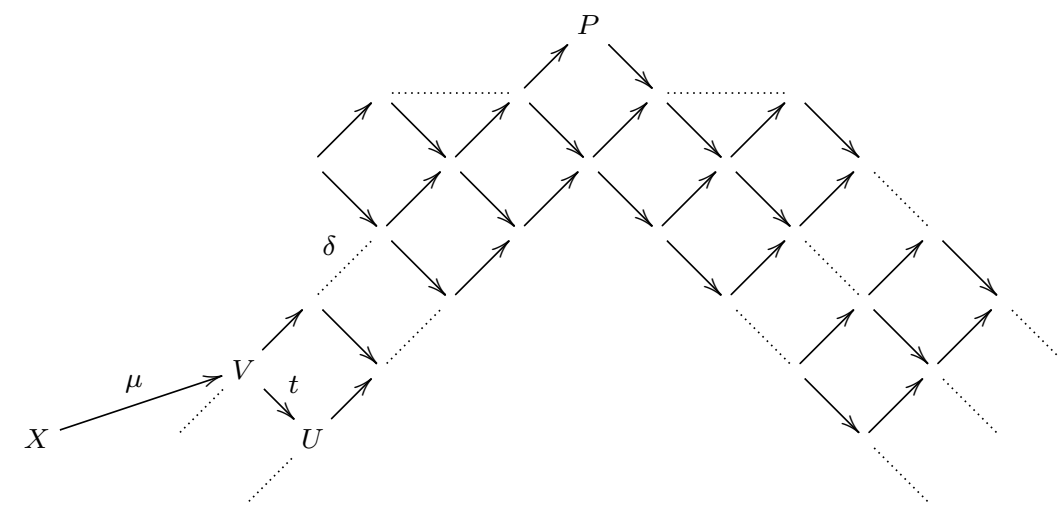

where $\delta: V \rightarrow P$ is a sectional path in $\Gamma$ and $P$ is a projective-injective module belonging to a mesh with exactly two middle terms. By inductive hypothesis we know that $\mu$ behaves well. The irreducible morphisms in $\delta$ have infinite left degree by [?]. Hence, $\delta \mu$ behaves well. Moreover, again by [?] since $t$ is an irreducible monomorphism then $d_{l}(t)=\infty$ and we get the result.

Then, it is enough to prove the result for zero paths in $\Gamma$, since if we have a non-zero path

$$
X_{1} \stackrel{f_{1}}{\longrightarrow} X_{2} \stackrel{f_{2}}{\longrightarrow} \cdots \stackrel{f_{n-1}}{\longrightarrow} X_{n} \stackrel{f_{n}}{\longrightarrow} X_{n+1}
$$


in $\Gamma$ then, as we see above, $f_{n} \ldots f_{1}$ behaves well, getting a contradiction with our assumption. Therefore, $f_{n} \ldots f_{1}=0$.

Now, any other composition of irreducible morphisms $h_{i}: X_{i} \rightarrow X_{i+1}$ for $i=1, \ldots, n$ is such that $h_{n} \ldots h_{1}=\delta f_{n} \ldots f_{1}+\mu$ with $\mu \in \Re_{A}^{n+1}\left(X_{1}, X_{n+1}\right)$ and $\delta \in \operatorname{Aut}\left(X_{n+1}\right)$. Hence, $h_{n} \ldots h_{1} \in \Re_{A}^{n+1}\left(X_{1}, X_{n+1}\right)$.

Assume that $h_{n} \ldots h_{1} \notin \Re_{A}^{\infty}\left(X_{1}, X_{n+1}\right)$, that is, the composition $h_{n} \ldots h_{1}$ belongs to $\Re_{A}^{m}\left(X_{1}, X_{n+1}\right) \backslash \Re_{A}^{m+1}\left(X_{1}, X_{n+1}\right)$ with $m>n$. Hence there is a non-zero path from $X_{1} \rightsquigarrow$ $X_{n+1}$ of length longest than $n$, contradicting Lemma ??. The proof is completed.

Note that as an immediate consequence of Proposition ?? and Theorem ?? we obtain Theorem A.

Our next two results are fundamental for the study of the composition of irreducible morphisms lying in a tube.

Lemma 3.16. Let $A$ be an artin algebra and $\Gamma$ a tube in $\Gamma_{A}$. Then

(a) If there is a zero path in $\Gamma$ from $X$ to $Y$ then any longest path in $\Gamma$ from $X$ to $Y$ vanishes.

(b) If there is a non-zero path $\gamma$ from $X$ to $Y$ in $\Gamma$ of length $m$ then $\operatorname{dp}(\gamma)=m$.

Proof. $(a)$. Let $A$ be an artin algebra and $\Gamma$ be a tube in $\Gamma_{A}$. From the definition of a tube we know that $\Gamma$ considered as a translation quiver can be obtained from a stable tube by an iterated application of admissible operations of type (ad 1) and ( $\left.\mathrm{ad} 1^{*}\right)$. Therefore, the statement follows from arguments similar to those applied in the proof of Lemma ??.

(b) Let $\gamma: X=X_{1} \rightarrow X_{2} \rightarrow \cdots \rightarrow X_{m} \rightarrow X_{m+1}=Y$ be a non-zero path in $\Gamma$ of length $m$. Then we have in $\Gamma$ the rectangle $\mathscr{S}\left(X, Z_{p}, Y, W\right)$ of the form

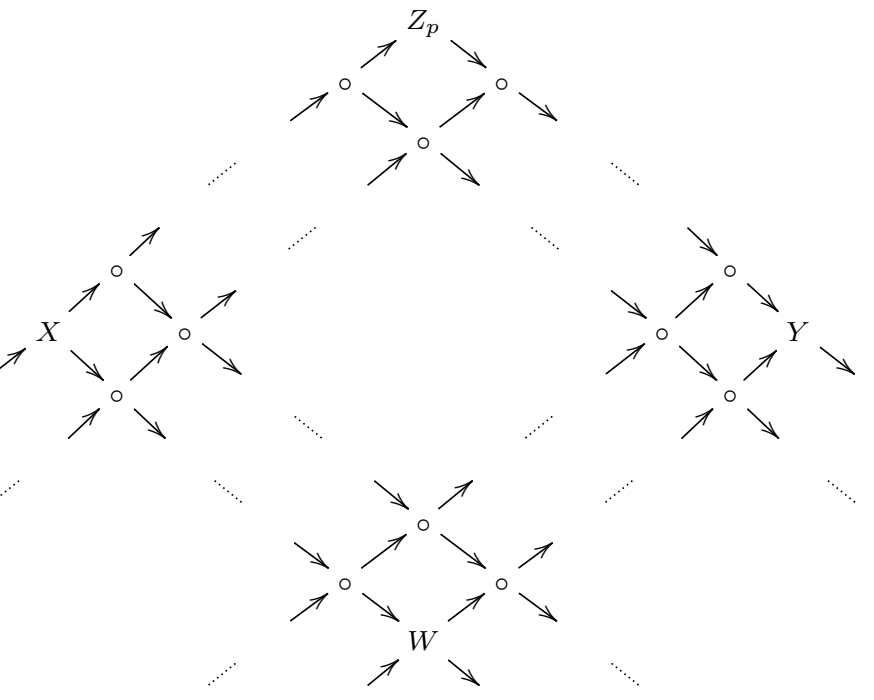

Observe that in this case any path in $\mathscr{S}\left(X, Z_{p}, Y, W\right)$ from $X$ to $Y$ is non-zero and has length $m$. Let $f: X \rightarrow Z_{p}$ be the composition of irreducible maps corresponding to the arrows of the sectional path $\alpha: X=X_{1} \rightarrow \cdots \rightarrow Z_{p}$, and let $g: Z_{p} \rightarrow Y$ be the composition of irreducible maps corresponding to the arrows of the sectional path $\beta: Z_{p} \rightarrow \cdots \rightarrow X_{m+1}=Y$. Since by [?, Section 1] the arrows of the path $\alpha$ (respectively, 
the path $\beta$ ) are of infinite right (respectively, left) degree, we infer that $g f \in \Re_{A}^{m}(X, Y) \backslash$ $\Re_{A}^{m+1}(X, Y)$. Hence $\operatorname{dp}(\gamma)=m$.

Lemma 3.17. Let $A$ be an artin algebra and $\Gamma$ a tube in $\Gamma_{A}$. Let $h_{i}: X_{i} \rightarrow X_{i+1}$ be irreducible morphisms with $X_{i} \in \Gamma$ for $i=1, \ldots, n+1$. If $0 \neq h_{n} \ldots h_{1} \in \Re_{A}^{n+1}\left(X_{1}, X_{n+1}\right)$ then there exists $f_{1}, \ldots, f_{n}$ such that $f_{n} \ldots f_{1}=0$ for any choice of irreducible morphisms $f_{i}: X_{i} \rightarrow X_{i+1}$ satisfying the mesh relations of $\Gamma$.

Proof. Consider irreducible morphisms $f_{i}: X_{i} \rightarrow X_{i+1}$ for $i=1, \ldots, n$ satisfying the mesh relations of $\Gamma$. By Lemma ?? we have that $h_{n} \ldots h_{1}=\delta f_{n} \ldots f_{1}+\mu$ with $\mu \in$ $\Re_{A}^{n+1}\left(X_{1}, X_{n+1}\right)$ and $\delta \in \operatorname{Aut}\left(X_{n+1}\right)$. Hence, $f_{n} \ldots f_{1} \in \Re_{A}^{n+1}\left(X_{1}, X_{n+1}\right)$.

Suppose that $f_{n} \ldots f_{1} \neq 0$. Then, by Lemma ?? (b), we get that $f_{n} \ldots f_{1}$ behaves well getting a contradiction with the fact that $f_{n} \ldots f_{1} \in \Re_{A}^{n+1}\left(X_{1}, X_{n+1}\right)$. Hence $f_{n} \ldots f_{1}=$ 0 .

Next, we prove one of our main results. We observe that the proof is similar to [?, Theorem A]. For the convenience of the reader we state it here.

Theorem 3.18. Let $A$ be an artin algebra and $\Gamma$ a tube in $\Gamma_{A}$. Let $h_{i}: X_{i} \rightarrow X_{i+1}$ be $n$ irreducible morphisms with $X_{i} \in \Gamma$ for $i=1, \ldots, n$. Then, $0 \neq h_{n} \ldots h_{1} \in \Re_{A}^{n+1}\left(X_{1}, X_{n+1}\right)$ if and only if $0 \neq h_{n} \ldots h_{1} \in \Re_{A}^{\infty}\left(X_{1}, X_{n+1}\right)$.

Proof. Assume that there are $n$ irreducible morphisms $h_{i}: X_{i} \rightarrow X_{i+1}$ such that $0 \neq$ $h_{n} \ldots h_{1} \in \Re_{A}^{n+1}\left(X_{1}, X_{n+1}\right)$. By Lemma ?? there are $n$ irreducible morphisms $f_{i}: X_{i} \rightarrow$ $X_{i+1}$ in the mesh satisfying that $f_{n} \ldots f_{1}=0$.

Suppose that $h_{n} \ldots h_{1} \in \Re_{A}^{n+k}\left(X_{1}, X_{n+1}\right) \backslash \Re_{A}^{n+k+1}\left(X_{1}, X_{n+1}\right)$, for some $k \geq 1$. By [?, V, Proposition 7.4] there is a non-zero path $\gamma: X_{1} \rightarrow X_{n+1}$ of irreducible morphisms of length $n+k$, whose composition does not belong to $\Re_{A}^{n+k+1}\left(X_{1}, X_{n+1}\right)$. Then, by Lemma ?? (a), we know that there is a zero path $\gamma^{\prime}: X_{1} \rightarrow X_{n+1}$ of length $n+k$ satisfying the mesh relations in $\Gamma$. By Lemma ?? we can write $\gamma^{\prime}=\delta \gamma+\mu$ with $\delta \in \operatorname{Aut}\left(X_{n+1}\right)$ and $\mu \in \Re_{A}^{n+k+1}\left(X_{1}, X_{n+1}\right)$. Hence, we conclude that $\gamma^{\prime} \in \Re_{A}^{n+k+1}\left(X_{1}, X_{n+1}\right)$ a contradiction.

The converse is clear.

\section{References}

[1] I. Assem, D. Simson, A. Skowroński, Elements of the Representation Theory of Associative Algebras 1: Techniques of Representation Theory, London Math. Soc. Stud. Texts, vol. 65, Cambridge Univ. Press, Cambridge, 2006.

[2] I. Assem, A. Skowroński, Minimal representation-infinite coil algebras, Manuscripta Math. 67 (1990), 305-331.

[3] I. Assem, A. Skowroński, Multicoil algebras, in: Representations of Algebras, Canad. Math. Soc. Conf. Proc., vol. 14, Amer. Math. Soc., Providence, RI, 1993, pp. 29-68.

[4] M. Auslander, I. Reiten, S. O. Smalø, Representation Theory of Artin Algebras, Cambridge Stud. in Adv. Math., vol. 36, Cambridge Univ. Press, Cambridge, 1995.

[5] R. Bautista, On irreducible maps, Bull. Amer. Math. Soc. (N. S.) 2 (1980), 177-180.

[6] J. Białkowski, K. Erdmann, A. Skowroński, Deformed preprojective algebras of generalized Dynkin type, Trans. Amer. Math. Soc. 359 (2007), 2625-2650.

[7] C. Chaio, Degrees and Composition of irreducible morphisms in almost presectional paths, Algebras and Representation Theory 17 (2014), no. 2, 407-432.

[8] C. Chaio, A generalization of the composition of irreducible morphisms in regular components, Algebras and Representation Theory (2014), DOI: 10.1007/s10468-014-9496-5. 
[9] C. Chaio, On the Harada and Sai bound, Bulletin of The London Math. Soc. 44, Issue 6 (2012), $1237-1245$.

[10] C. Chaio, S. Liu, A note on the radical of a module category, Communications in Algebra 41 (2013), no. $12,4419-4424$.

[11] C. Chaio, F. U. Coelho, S. Trepode, On the composite of two irreducible morphisms in radical cube, J. Algebra 312 (2007) 650-667.

[12] C. Chaio, F. U. Coelho, S. Trepode, On the composite of irreducible morphisms in almost sectional paths, J. Pure Appl. Algebra 212 (2008), 244-261.

[13] C. Chaio, F. U. Coelho, S. Trepode, On the composite of three irreducible morphisms in the fourth power of the radical, Comm. Algebra 39 (2011), 555-559.

[14] C. Chaio, P. Malicki and A. Skowroński, Degrees of irreducible morphisms in coherent almost cyclic Auslander-Reiten components, J. Algebra 391, Issue 10 (2013), 125-151.

[15] C. Chaio, P. Le Meur, S. Trepode, Degrees of irreducible morphisms and finite-representation type, J. London Math. Soc. (2) 84 (2011), 35-57.

[16] C. Chaio, S. Trepode, The composite of irreducible morphisms in standard components, J. Algebra 323 (2010), 1000-1011.

[17] G. D'Este, C. M. Ringel, Coherent tubes, J. Algebra 87 (1984), 150-201.

[18] K. Erdmann, Blocks of Tame Representation Type and Related Algebras, Lect. Notes in Math. Vol 1428, Berlin-New York: Springer- Verlag 1990.

[19] K. Erdmann, On tubes for blocks of wild type, Colloq. Math. 82 (1999), 261-270.

[20] K. Igusa, G. Todorov, A characterization of finite Auslander-Reiten quivers, J. Algebra 89 (1984), 148-177.

[21] H. Lenzing, A. Skowroński, Selfinjective algebras of wild canonical type, Colloq. Math. 96 (2003), 245-275.

[22] S. Liu, Degrees of irreducible maps and the shapes of the Auslander-Reiten quivers, J. London Math. Soc. 45 (1992), 32-54.

[23] P. Malicki, Coherent quasi-tubes, Bull. Polish Acad. Sci. Math. 44 (1996), 461-471.

[24] P. Malicki, Generalized coil enlargements of algebras, Colloq. Math. 76 (1998), 57-83.

[25] P. Malicki, J. A. de la Peña, A. Skowroński, Finite cycles of indecomposable modules, J. Pure Appl. Algebra 219 (2015), 1761-1799.

[26] P. Malicki, A. Skowroński, Almost cyclic coherent components of an Auslander-Reiten quiver, J. Algebra 229 (2000), 695-749.

[27] P. Malicki, A. Skowroński, Algebras with separating almost cyclic coherent Auslander-Reiten components, J. Algebra 291 (2005), 208-237.

[28] P. Malicki, A. Skowroński, On the additive categories of generalized standard almost cyclic coherent Auslander-Reiten components, J. Algebra 316 (2007), 133-146.

[29] P. Malicki, A. Skowroński, On the indecomposable modules in almost cyclic coherent AuslanderReiten components, J. Math. Soc. Japan 63 (2011), 1121-1154.

[30] P. Malicki, A. Skowroński, Algebras with separating Auslander-Reiten components, in: Representations of Algebras and Related Topics, European Math. Soc. Series Congress Reports, European Math. Soc. Publ. House, Zürich, 2011, 251-353.

[31] P. Malicki, A. Skowroński, On the number of simple and projective modules in the quasi-tubes of selfinjective algebras, Comm. Algebra 39 (2011), no. 1, 322-334.

[32] P. Malicki, A. Skowroński, Hochschild cohomology of generalized multicoil algebras, Colloq. Math. 136 (2014), 231-254.

[33] C. M. Ringel, Tame Algebras and Integral Quadratic Forms, Lecture Notes in Math., vol. 1099, Springer-Verlag, Berlin-Heidelberg, 1984.

[34] A. Skowroński, Selfinjective algebras: finite and tame type. Trends in Representation Theory of Algebras and Related Topics. Contemp. Math. Vol. 406. Providence: Amer. Math. Soc., 2006, pp. 169-238.

[35] Y. Zhang, The structure of stable components, Canad. J. Math. 43 (1991), 652-672. 